\title{
QUANTITATIVE RELATIVE COMPARISON OF CFD SIMULATION UNCERTAINTIES FOR A TRANSONIC DIFFUSER PROBLEM
}

\author{
Serhat Hosder*, Bernard Grossman, Raphael T. Haftka, \\ William H. Mason, and Layne T. Watson \\ Multidisciplinary Analysis and Design (MAD) Center for Advanced Vehicles \\ Virginia Polytechnic Institute and State University \\ Blacksburg, VA 24061-0203, USA
}

\begin{abstract}
Different sources of uncertainty in CFD simulations are illustrated by a detailed study of two-dimensional, turbulent, transonic flow in a converging-diverging channel. Runs were performed with the commercial CFD code GASP using different turbulence models, grid levels, and flux-limiters to see the effect of each on the CFD simulation uncertainties. Two flow conditions were studied by changing the exit pressure ratio: the first is a complex case with a strong shock and a separated flow region, the second is the weak shock case with no separation. The uncertainty in CFD simulations has been studied in terms of four contributions: (1) discretization error, (2) error in geometry representation, (3) turbulence model, and (4) the downstream boundary condition. In this paper, we

\footnotetext{
${ }^{*}$ Corresponding author. Department of Aerospace and Ocean Engineering, 215 Randolph Hall, Virginia Polytechnic Institute and State University, Blacksburg, VA 24061-0203, USA. Phone: 1-540-2307717, Fax: 1-540-2319632, e-mail: shosder@vt.edu
} 
have quantified the relative contribution and the importance of each source of uncertainty and shown the level of scatter in results that a well informed CFD user may obtain in a typical design activity. The nozzle efficiency results obtained in this study showed that the range of variation for the strong shock case was much larger than that observed in the weak shock case. The discretization errors were up to $6 \%$ and the relative uncertainty originating from the selection of different turbulence models was as large as $9 \%$ for the strong shock case. Furthermore, the results demonstrated that grid convergence is not achieved with grid levels that have moderate mesh sizes and showed that highly refined grids are required to obtain solutions with an acceptable level of accuracy in design problems that involve simulations of complex flow fields. The results illustrated the interaction of different sources of uncertainty and showed that the magnitudes of numerical errors are influenced by the physical models used.

Keywords: CFD based design, uncertainty, error, multidisciplinary design optimization

\section{INTRODUCTION}

Computational fluid dynamics (CFD) has become an important aero/hydrodynamic analysis and design tool in recent years. CFD simulations with different levels of fidelity, ranging from linear potential flow solvers to full Navier-Stokes codes, are widely used in the multidisciplinary design and optimization (MDO) of advanced aerospace and ocean vehicles [1]. Although low-fidelity CFD tools have low computational cost and are eas- 
ily used, the full viscous equations are needed for the simulation of complex turbulent separated flows, which occur in many practical cases such as high-angle-of attack flight, high-lift devices, maneuvering submarines and missiles [2]. Even for cases when there is no flow separation, the use of high-fidelity CFD simulations is desirable for obtaining higher accuracy. Due to modeling, discretization, and computation errors, the results obtained from CFD simulations have a certain level of uncertainty. It is important to understand the sources of CFD simulation errors and their magnitudes to be able to assess the magnitude of the uncertainty in the results.

The results presented in the $1^{\text {st }}[3,4]$ and the $2^{\text {st }}$ AIAA CFD Drag Prediction Workshops [5] illustrate the importance of understanding the uncertainty and its sources in CFD simulations. As an example, in the $1^{\text {st }}$ Drag Prediction Workshop, many of the performance quantities of interest for the DLR-F4 wing-body configuration (workshop test case), such as the lift curve slope, the drag polar, or the drag rise Mach number, obtained from the CFD solutions of 18 different participants using different codes, grid types, and turbulence models, showed a large variation, which revealed the general issue of accuracy and credibility in CFD simulations.

The objective of this work is to illustrate different sources of uncertainty and their interactions in CFD simulations, by a careful study of a typical, but complex, fluid dynamics problem. We compare the magnitude of each source of uncertainty and its importance for design. It should be noted that this is not a paper on validation or verification. Such studies have been conducted for the CFD code used here. (See Neel et al. [6] and 
Brown [7].) The experimental results are included only for reference and are not used for the purpose of code validation. In this study, we (1) quantify the relative contribution and the importance of each source of uncertainty and show the level of scatter in results that a well informed CFD user may obtain in a design or analysis activity, (2) illustrate the interaction of different sources of uncertainty, and (3) show that the complex flow fields with separated flow regions require highly refined grids to obtain solutions with an acceptable level of accuracy.

The problem studied in this paper is a two-dimensional, turbulent, transonic flow in a converging-diverging channel. CFD calculations were done with the General Aerodynamic Simulation Program (GASP) [8]. Runs were performed with different turbulence models, grid densities, and flux-limiters to see the effect of each on the CFD simulation uncertainties. In this paper, we focus on four sources of uncertainty: (1) discretization error, (2) error in geometry representation, (3) turbulence model, and (4) change in the downstream boundary condition.

\section{UNCERTAINTY SOURCES}

To better understand the accuracy of CFD simulations, the main sources of errors and uncertainties should be identified. Oberkampf and Blottner [9] classified CFD error sources. In their classification, the error sources are grouped under four main categories: (1) physical modeling errors, (2) discretization errors, (3) programming errors, and (4) 
solution errors.

Physical modeling errors originate from the inaccuracies in the mathematical models of the physics. The errors in the partial differential equations (PDEs) describing the flow, the auxiliary (closure) physical models, and the boundary conditions for all the PDEs are included in this category. Turbulence models used in viscous calculations are considered as one of the auxiliary physical models, usually the most important one. They are used for modeling the additional terms that originate as the result of Reynolds averaging, which in itself is a physical model.

Oberkampf and Blottner [9] define discretization errors as the errors caused by the numerical replacement of PDEs, the auxiliary physical models and continuum boundary conditions by algebraic equations. Consistency and the stability of the discretized PDEs, spatial (grid) and temporal resolution, errors originating from the discretization of the continuum boundary conditions are listed under this category. The difference between the exact solution to the discrete equations and the approximate (or computer) solution is defined as the solution error of the discrete equations. Iterative convergence error of the steady-state or the transient flow simulations is included in this category. A similar description of the discretization errors can also be found in Roache [10, 11], and Pelletier et al. [12].

Since the terms error and uncertainty are commonly used interchangeably in many CFD studies, it will be useful to give a definition for each. Uncertainty, itself, can be defined in many forms depending on the application field as listed in DeLaurentis and Mavris [13]. 
For computational simulations, Oberkampf et al. $[14,15]$ described uncertainty as a potential deficiency in any phase or activity of the modeling process that is due to the lack of knowledge, whereas error is defined as a recognizable deficiency in any phase or activity of modeling and simulation.

Considering these definitions, any deficiency in the physical modeling of the CFD activities can be regarded as uncertainty (such as uncertainty in the accuracy of turbulence models, uncertainty in the geometry, uncertainty in thermophysical parameters, etc.), whereas the deficiency associated with the discretization process can be classified as error [15].

Discretization errors can be quantified by using methods like Richardson extrapolation or the grid convergence index (GCI), a method developed by Roache [11] for uniform reporting of grid convergence studies. However, these methods require fine grid resolution in the asymptotic range, which may be hard to achieve in the simulation of flow fields around complex geometries. Also, non-monotonic grid convergence, which may be observed in many flow simulations, prohibits or reduces the applicability of such methods. That is, it is often difficult to estimate errors in order to separate them from uncertainties. Therefore, for the rest of the paper, the term uncertainty will be used to describe the inaccuracy in the CFD solution variables originating from discretization, solution, or physical modeling errors. The combined treatment of CFD simulation uncertainties and errors was also presented in studies by Roache $[16,11]$. 


\section{TRANSONIC DIFFUSER CASE}

\subsection{Description of the physical problem}

The test case presented in this paper is the simulation of a 2-D, turbulent, transonic flow in a converging-diverging channel, known as the Sajben Transonic Diffuser in CFD studies [17]. The exit station is at $x / h_{t}=8.65$ for the geometry shown at the top part of Figure 1, where $h_{t}$ is the throat height. This is the original geometry used in the computations and a large portion of the results with different solution and physical modeling parameters are obtained with this version. The exit station is located at $x / h_{t}=$ 14.44 for the other geometry shown in Figure 1. This extended geometry is used to study the effect of varying the downstream boundary location on the CFD simulation results.

For both geometries, the bottom wall of the channel is flat and the converging-diverging section of the top wall is described by an analytical function of $x / h_{t}$ defined in Bogar et al. [18]. In addition to these two geometries, a third version of the same diffuser (the modified-wall geometry) has been developed for this research and has been used in our calculations. This version has the same inlet and exit locations as the original geometry, but the upper wall is described by natural cubic splines fitted to the geometric data points that were measured in the experimental studies. The upper wall contour obtained by the analytical equation and the contour described by experimental data points are slightly different, which may be due the error in fabrication and the measurement of the fabricated geometry. Note that if there were no measurement errors, the experimental 
data points would be the representative of the "as made" hardware actually used in the experiment. In our studies, we use the modified-wall geometry to find the effects of geometric uncertainty on the numerical results.

Despite the relatively simple geometry, the flow has a complex structure. The exit pressure ratio $P_{e} / P_{0 i}$ sets the strength and the location of a shock that appears downstream of the throat (Figure 2). In our studies, for the original and the modified-wall geometries, we define $P_{e} / P_{0 i}=0.72$ as the strong shock case and $P_{e} / P_{0 i}=0.82$ as the weak shock case. A separated flow region exists just after the shock at $P_{e} / P_{0 i}=0.72$. Although a nominal exit station was defined at $x / h_{t}=8.65$ for the diffuser used in the experiments, the physical exit station is located at $x / h_{t}=14.44$. The geometry used in the experiments has a rectangular cross section with the aspect ratio (height/width) of 0.35 at the constant-area region upstream of the throat, 0.25 at the throat, and 0.38 at the constant-area region downstream of the throat. In the experiments, $P_{e} / P_{0 i}$ was measured as 0.7468 and 0.8368 for the strong and the weak shock cases respectively at the physical exit location. Table 1 gives a summary of the different versions of the transonic diffuser geometry and exit pressure ratios used in our computations.

\subsection{Computational modeling}

CFD calculations were performed with GASP, a Reynolds-averaged, three-dimensional, finite-volume, Navier-Stokes code, which is capable of solving steady state (time asymp- 
totic) and time dependent problems. For this problem, the inviscid fluxes were calculated by an upwind-biased third order spatially accurate Roe flux scheme. The minimum modulus (Min-Mod) and Van Albada's flux limiters were used to prevent non-physical oscillations in the solution. All the viscous terms were included in the solution and two turbulence models, Spalart-Allmaras [19] (Sp-Al) and $k$ - $\omega$ [20] (Wilcox, 1998 version) with Sarkar's Compressibility Correction, were used for modeling the viscous terms. Note that all solutions presented in this paper were obtained with version 4.1.0 of the GASP code compiled for SGI machines that run IRIX $^{\circledR} 6.5$ UNIX $^{\circledR}$ operating system.

It should be noted that the actual flow in the diffuser geometry is three-dimensional. In our study, we treat the flow as two-dimensional. Based on this assumption, we study different uncertainty sources for relative comparison. Since this work is not a validation study, we do not compare the numerical results with the measurements. However, if one wants to compare the CFD results with the experiment in a validation study, the uncertainty due to the two-dimensional modeling of a three-dimensional flow should also be taken into account, especially in evaluating the accuracy of turbulence models. The actual geometry used in the experiments has suction slots placed at $x / h_{t}=9.8$ on the bottom and the side walls to limit the growth of the boundary layer. These suction slots should also be modeled in a validation study if one wants to compare the numerical results obtained downstream of the slots with the experimental data.

The adiabatic no-slip boundary condition was used on the top and the bottom walls of the transonic diffuser geometry. At the inlet, a constant total pressure $\left(P_{0 i}\right)$ and 
temperature $\left(T_{0 i}\right)$ were specified (subsonic $P_{0 i}-T_{0 i}$ inflow boundary condition in GASP). The static pressure was taken from the adjacent interior cell and the other flow variables were calculated by using isentropic relations. At the exit, the outflow boundary was set to a constant static pressure $\left(P_{e}\right)$, while the remaining flow variables were extrapolated from the interior cells. To initialize each CFD solution, inflow conditions were used.

The iterative convergence of each case to a steady-state solution was examined by monitoring the overall residual, which is the sum (over all the cells in the computational domain) of the $L^{2}$ norm of all the governing equations solved in each cell. In addition to this overall residual information, the individual residual of each equation and some of the output quantities were also monitored.

The sizes and the nomenclature of the grids used in the computations are given in Table 2. Grid 2 (top) and Grid $2_{\text {ext }}$ (bottom) are shown in Figure 1. The details about the grids used in the computations can be found in Hosder et al. [21].

\section{RESULTS AND DISCUSSION}

For the transonic flow in the converging-diverging channel, the uncertainty of the CFD simulations is investigated by examining the nozzle efficiency $\left(n_{e f f}\right)$ as a global output quantity obtained at different $P_{e} / P_{0 i}$ ratios with different grids, flux limiters (Min-Mod and Van Albada), and turbulence models (Sp-Al and $k-\omega)$. The nozzle efficiency is defined 


$$
n_{e f f}=\frac{H_{0 i}-H_{e}}{H_{0 i}-H_{e s}}
$$

where $H_{0 i}$ is total enthalpy at the inlet, $H_{e}$ the enthalpy at the exit, and $H_{e s}$ the exit enthalpy at the state that would be reached by isentropic expansion to the actual pressure at the exit. Since the enthalpy distribution at the exit was not uniform, $H_{e}$ and $H_{e s}$ were obtained by integrating the cell-averaged enthalpy values across the exit plane. Figure 3 shows the nozzle efficiencies obtained with different grid levels, turbulence models, limiters, geometries, and boundary conditions for the strong and the weak shock cases.

In our studies on the transonic diffuser case, we have focused on five sources of the CFD simulation uncertainties: (1) iterative convergence error, (2) discretization error, (3) error in geometry representation, (4) turbulence model, and (5) change in the downstream boundary condition. In general, (1) and (2) contribute to the numerical uncertainty, which is the subject of the verification process; (3), (4), and (5) contribute to the physical modeling uncertainty, which is the concern of the validation process. In this paper, the iterative convergence error is not included. In our studies, we have seen that the contribution of the iterative convergence error to the overall uncertainty is negligible. For a detailed analysis of the iterative convergence error in the transonic diffuser case, the reader should refer to Hosder et al. [21]. It should be noted again that the current work is not a validation study. By studying uncertainty sources (3), (4), and (5), we would like to investigate the relative contribution and the importance of each source of uncertainty and show the level of scatter in results that a well informed CFD user may 
obtain in a design process. In a proper validation study, measurements from experiments should be compared to the numerical results to determine the accuracy of physical models, which requires detailed information about the uncertainties in measurements. For our test case, the experimental data may contain uncertainties originating from many factors such as geometric irregularities, difference between the actual $P_{e} / P_{0 i}$ and its intended value, measurement errors, heat transfer to the fluid, etc. Since such uncertainties for the current experimental data set are not available, we will not compare our results with the experiment for the purpose of validation. The experimental results will be included for reference only.

In our discussion, we will first focus on the discretization errors, then the uncertainties originating from geometry representation and the change in downstream boundary condition. Finally, we will make relative comparisons of each source of uncertainty. This will also include the relative uncertainty due to the selection of turbulence models.

\subsection{The Discretization Error}

The grid level and the flux-limiter affect the magnitude of the discretization error. Grid level determines the spatial resolution, and the limiter is part of the discretization scheme, which reduces the spatial accuracy of the method to first order in the vicinity of shock waves. In this paper, Richardson extrapolation technique has been used to estimate the magnitude of the discretization error at each grid level for cases that show monotonic 
convergence. This method is based on the assumption that $f_{k}$, a local or global output variable obtained at grid level $k$, can be represented by

$$
f_{k}=f_{\text {exact }}+\alpha h^{p}+O\left(h^{p+1}\right),
$$

where $h$ is a measure of grid spacing, $p$ the order of the method, and $\alpha$ the $p^{\text {th }}$-order error coefficient. Note that Equation 2 will be valid when $f$ is smooth and in the asymptotic grid convergence range. In most cases, the observed order of spatial accuracy is different than the nominal (theoretical) order of the numerical method due to factors such as the lack of sufficient grid convergence, existence of discontinuities in the solution domain, boundary condition implementation, flux-limiters, etc. Therefore, the observed value of $p$ should be determined and used in the calculations required for approximating $f_{\text {exact }}$ and the discretization error. Calculation of the observed order of accuracy $(\tilde{p})$ needs the solutions from three grid levels, and the estimate of the $f_{\text {exact }}$ value requires two grid levels. The details of the calculations are given in Appendix A. As an alternative to Richardson extrapolation, some other methods can also be used to asses the solution accuracy of the CFD simulations. Recently, Vaidyanathan et al. [22] used a least square extrapolation method to project the numerical solutions of sample Navier-Stokes computations from multiple, coarser base grids onto a finer grid for improving the solution accuracy by minimizing the residual of the discretized governing equations over the projected grid. Their work on sample problems demonstrated the potential of the least square extrapolation method as a quantitative measure for accuracy improvement that can also be used for code verification. 
Table 3 summarizes the discretization error in nozzle efficiency results obtained with the original geometry. For each case with a different turbulence model, limiter, and exit pressure ratio, the approximation to the exact value of nozzle efficiency is denoted by $\left(\tilde{n}_{\text {eff }}\right)_{\text {exact }}$ and the discretization error at a grid level $k$ is calculated by

$$
\operatorname{error}(\%)=\left|\frac{\left(n_{e f f}\right)_{k}-\left(\tilde{n}_{\text {eff }}\right)_{\text {exact }}}{\left(\tilde{n}_{\text {eff }}\right)_{\text {exact }}} \times 100\right|
$$

The following gives a summary of the discretization error results obtained with different turbulence models and limiters.

\subsubsection{Sp-Al model and Van Albada limiter}

The grid convergence is monotonic both for the strong and the weak shock cases. At grid level g2, the discretization error is $4.5 \%$ for the strong shock case and $1.5 \%$ for the weak shock case.

\subsubsection{Sp-Al model and Min-Mod limiter}

The grid convergence is monotonic both for the strong and the weak shock cases. For the strong shock case, the discretization error is equal to $6.8 \%$ at grid level g2, which is the largest of errors obtained at this grid level. The discretization error is calculated as $3.5 \%$ at the same grid level for the weak shock case. The smallest observed order of accuracy $(\tilde{p}=1.2)$ is obtained with this turbulence model and limiter for the strong shock case.

In addition to the results presented in Table 3, the finest grid level (g5) was used only in 
the discretization error analysis of the strong shock case obtained with this turbulence model and limiter. Table 6 in Appendix A gives the discretization error results obtained using grid level g5.

\subsection{3 $k-\omega$ model and Van Albada limiter}

The monotonic grid convergence is observed only for the weak shock case and the discretization error is equal to $1.5 \%$ at grid level g2. The largest observed order of accuracy $(\tilde{p}=1.98)$ is obtained with this turbulence model and the limiter.

\subsection{4 $k-\omega$ model and Min-Mod limiter}

The monotonic grid convergence is achieved again only for the weak shock case. A discretization error of $1.5 \%$ is obtained at grid level g2. For the weak shock case, the $k-\omega$ turbulence model gives lower discretization errors compared to the $\mathrm{Sp}-\mathrm{Al}$ results at the same grid level, regardless of the limiter used.

\subsubsection{General remarks}

Figures 4 and 5 give a graphical representation of the results presented in Table 3 . In these figures, the discretization errors are added as error bars over the $\left(\tilde{n}_{\text {eff }}\right)_{\text {exact }}$ values for each turbulence model and the limiter at each grid level. One can see that the discretization error magnitudes are different for cases with different turbulence models when results 
obtained with the same limiter and grid level are compared at each shock condition. This indicates the effect of the turbulence model on discretization errors and implies that the magnitudes of numerical errors are influenced by the physical models used. Figures 4 and 5 also show that only grid levels g3 and g4 make it possible to discriminate between turbulence models, if one wants to analyze the difference in the results that come purely from physical modeling uncertainties. Although the discretization errors originating from spatial resolution and the flux limiter are closely coupled, it can be seen that the relative uncertainty due to the choice of the limiter is more significant for the strong shock case. For both pressure ratios, the nozzle efficiency values obtained with different limiters become closer to each other as the mesh is refined.

The discretization error analysis of nozzle efficiency results also shows that grid convergence is not achieved with grid levels that have moderate mesh sizes, especially for the strong shock case. When the flow-field includes shocks with substantial flow separation, highly refined grids, which are beyond the grid levels we use in this study, may be needed for spatial convergence. Even with the finest mesh level we could afford, achieving asymptotic convergence is not certain. Note that a single solution on Grid $5(640 \times 400$ cells $)$, the finest mesh used in this study, required approximately 1170 hours of total node CPU time on a SGI Origin2000 with six processors, when 10000 cycles were run with this grid in year 2002 .

At the same grid level, the discretization errors are smaller for the weak shock cases compared to the strong shock results. This observation indicates the effect of flow structure 
complexity on grid convergence. Besides nozzle efficiency, a global solution parameter, one can also see this effect by examining local solution quantities. When we look at the Mach number at two points in the original geometry, one upstream of the shock $\left(x / h_{t}=-1.5\right)$ and the other downstream of the shock $\left(x / h_{t}=8.65\right.$, the exit plane $)$, both of which are located at the mid point of the local channel heights (Figure 6), we see the convergence of the Mach number upstream of the shock for all the cases. However, for the strong shock case, the lack of convergence downstream of the shock at all grid levels with the $k-\omega$ model can be observed. For the Sp-Al case, we see the convergence only at grid levels g3 and g4. For the weak shock case, downstream of the shock, the convergence at all grid levels with the $k-\omega$ model is also seen. At this pressure ratio, Sp-Al model results do not seem to converge, although the difference between each grid level is small. These results again indicate the effect of the complex flow structure downstream of the shock, especially the separated flow region seen in the strong shock case, on the grid convergence.

In Table 3, the observed order of accuracy $\tilde{p}$ is smaller than the nominal order of the scheme and its value is different for each case with a different turbulence model, limiter, and shock condition. The values of both $\left(\tilde{n}_{\text {eff }}\right)_{\text {exact }}$ and $\tilde{p}$ also depend on the grid levels used in their calculations. For example, the $\tilde{p}$ value was calculated as 1.322 and 1.849 for the Sp-Al, Min-Mod, strong shock case with different grid levels (see Appendix A, Table 6). The difference in $\tilde{p}$ value due to the grid levels used in its calculation may degrade the accuracy of the discretization error approximation with Richardson 
extrapolation. One should also note the fact that the flow problem studied here contains a physical discontinuity in the form of a shock wave. Due to flux-limiting, the order of the method is reduced across the shock and the numerical method can exhibit the characteristics of a mixed-order scheme. The detailed studies on mixed-order schemes by Casper and Carpenter [23] and Roy [24] show that such methods may have two asymptotic regions, each having a different order. On coarser mesh levels one can see the nominal order of the method in grid convergence, whereas a first-order asymptotic region can be observed on sufficiently refined meshes. This complex behavior of mixed-order methods can cause the difference between the observed order and the nominal order of the method, and also non-monotonic grid convergence in some cases such as the strong shock, k- $\omega$ results obtained in our study (See Figure 3).

Figure 7 shows another effect of the discretization error on design, namely the numerical noise. In this figure, the noisy behavior of the nozzle efficiency results obtained with Grid 1 can be seen for both turbulence models. The order of the noise error is much smaller than the error between each grid level, however, this can be a significant source of uncertainty if the results of Grid 1 are used in a gradient based optimization.

\subsection{Error in the Geometry Representation}

The contribution of the error in geometry representation to CFD simulation uncertainties is studied by comparing the results of the modified-wall and the original geometry 
obtained with the same turbulence model, limiter, and grid level. Figure 8 gives the percent error distribution in $y / h_{t}$ (difference from the analytical value) for the upper wall of the modified-wall geometry at the data points measured in the experiments. A natural cubic spline interpolating these data points was used to obtain the upper wall contour. The difference between the upper wall contours of the original and the modified-wall geometry in the vicinity of the throat location is shown in Figure 9. Note that since we do not have enough information about the uncertainty in the measured experimental data, the CFD results obtained with the modified-wall geometry will not be compared to the experimental data. The experimental wall pressure values will be included in Figures 10 and 11 only for reference. Here, the modified-wall geometry is created to study the relative uncertainty in the results due to the error in geometry representation. The nozzle efficiency values obtained with the modified-wall geometry will be compared to the original geometry results in Section 4.4 .

We can make the following observations about the effect of geometry error on the upperwall static pressure. The flow becomes supersonic just after the throat and is very sensitive to the geometric irregularities for both $P_{e} / P_{0 i}=0.72$ and 0.82 . From the top wall pressure distributions shown in Figures 10 and 11, a local expansion/compression region can be seen around $x / h_{t}=0.5$ with the modified-wall geometry. This is due to the local bumps created by two experimental data points, the third and the fifth ones from the throat (Figure 9). Since neither the wall pressure results obtained with the original geometry nor the experimental values have this local expansion/compression, 
the values of these problematic points may contain some measurement error. The locations of these two points were modified by moving them in the negative $y$-direction halfway between their original value and the analytical equation value obtained at the corresponding $x / h_{t}$ locations. These modified locations are shown with black circles in Figure 9. The wall pressure results of the geometry with the modified experimental points (Figures 10 and 11) show that the local expansion/compression region seems to be smoothed, although not totally removed.

\subsection{Downstream Boundary Condition}

The effect of the downstream boundary location variation on the CFD simulation results of the transonic diffuser case has been investigated using the extended geometry, which has the physical exit station at the same location as the geometry used in the actual experiments. The runs were performed only with the Sp-Al model and the Van Albada limiter. Two $P_{e} / P_{0 i}$ ratios were used for the strong shock case: 0.72 and 0.7468 . For the weak shock case, $P_{e} / P_{0 i}$ ratios were 0.82 and 0.8368 . For each case, the second pressure ratio is the same value measured at the physical exit station of the geometry used in the experiments. The nozzle efficiency results obtained with the extended geometry and different $P_{e} / P_{0 i}$ ratios will be compared to the original geometry results in Section 4.4 to determine the relative uncertainty due to the change in downstream boundary condition.

We can examine the streamline pattern and the wall pressures to study the effect of the 
downstream boundary condition on the flow structure. Figure 12 shows the streamline patterns of the separated flow region obtained with different geometries and $P_{e} / P_{0 i}$ ratios in the strong shock case. The comparison of the separation bubble size is given in Figure 13. The separation bubble obtained with the extended geometry and $P_{e} / P_{0 i}=$ 0.72 is bigger and extends farther in the downstream direction compared to the other two cases. The separation bubbles obtained with the original geometry $\left(P_{e} / P_{0 i}=0.72\right)$ and the extended geometry $\left(P_{e} / P_{0 i}=0.7468\right)$ are approximately the same in size. These results are also consistent with the top wall pressure distributions given in Figure 14. Here experimental wall static pressures are again shown for reference only.

With the extended geometry and $P_{e} / P_{0 i}=0.72$, the flow accelerates more under the separation bubble, and the pressure is lower compared to the other cases where the separation bubbles have smaller thickness. Moving the exit location further downstream increases the strength of the shock and the size of the separation region. As the shock gets stronger, its location is shifted downstream. On the other hand, increasing $P_{e} / P_{0 i}$ reduces the strength of the shock, and moves the shock location upstream.

\subsection{Relative Comparison of Different Uncertainty Sources}

We use nozzle efficiency as a global indicator of the CFD results in the transonic diffuser case and the scatter in the computed values of this quantity originates from the use of different grid levels, limiters, turbulence models, geometries, and boundary conditions 
for each shock strength case. A graphical representation of this variation is given in Figure 3. This figure shows a cloud of results that a reasonably informed user may obtain from CFD calculations. The numerical value of each point is presented in Table 4 . We will analyze the scatter in nozzle efficiency results starting from grid level 2 , since the coarse Grid 1 will not be used by those that have significant experience in performing CFD simulations. We will examine the results of grid levels 2,3 , and 4 for the relative comparison of the uncertainty sources assuming that all these grid levels are used by different CFD engineers for the design of a transonic diffuser.

For the purpose of determining the variation in nozzle efficiency in terms of a percent value, we use the g4, Sp-Al, Van-Albada result as the comparator. When we consider the cases obtained with the original geometry, maximum variation for the strong shock condition is $9.9 \%$ and observed between the results of g2, $k-\omega$, Min-Mod and g4, Sp-Al, and Van Albada. Maximum difference in the weak shock results is $3.8 \%$ and obtained between the results of g2, $k-\omega$, Van Albada and g4, Sp-Al, and Min-Mod.

For each case with a different turbulence model and limiter, the variation between the results of g2 and g4 may be used to get an estimate of the uncertainty due to discretization error. The maximum variation for the strong shock is $5.7 \%$ and obtained with Sp-Al model and the Min-Mod limiter. For the weak shock case, the maximum difference is $3.5 \%$ and obtained with the same turbulence model and limiter.

We can approximate the relative uncertainty originating from the selection of different turbulence models by comparing the nozzle efficiency values obtained with the same 
limiter and the grid level. At grid level 4, the maximum difference between the strong shock results of Sp-Al and $k-\omega$ model is $9.2 \%$ and obtained with the Min-Mod limiter. For the weak shock case, the maximum difference at grid level 2 is $2.2 \%$, and obtained with the same limiter. It should be noted that, at each grid level, relative uncertainty due to the turbulence models is different resulting from the interaction of physical modeling uncertainties with the numerical errors.

For the strong shock case, at each grid level, the difference between nozzle efficiency values of the original geometry and the results of the modified-wall geometry is much smaller than the variations originating from the other sources of uncertainty regardless of the turbulence model and the limiter used. On the other hand, this difference is notable for the weak shock case and varies between $0.9 \%$ and $1.4 \%$.

Nozzle efficiency values of the extended geometry show considerable deviation from the results of the original geometry at certain grid levels, when 0.7468 and 0.8368 are used as the exit pressure ratios for the strong and the weak shock cases, respectively. For the exit pressure ratio of 0.7468 , the maximum difference is $1.8 \%$ and obtained with grid level 3. The maximum difference for the exit pressure ratio of 0.8368 is $6.9 \%$ and observed at grid level 4. The difference between the results of the original and the extended geometry is smaller when the exit pressure ratios of 0.72 and 0.82 are used. For the exit pressure ratio of 0.72 , the maximum difference is $0.8 \%$ and observed at grid level 3. A maximum difference of $1.1 \%$ is obtained at grid level 2 for the exit pressure ratio of 0.82 .

Principal observations on uncertainties in nozzle efficiencies are summarized in Table 5. 


\section{CONCLUSIONS}

Different sources of uncertainty in CFD simulations were illustrated by examining a 2-D, turbulent, transonic flow in a converging-diverging channel at various exit pressure ratios using the commercial CFD code GASP. Runs were performed with different turbulence models (Sp-Al and $k-\omega$ ), grid levels, and flux-limiters (Min-Mod and Van Albada). Two flow conditions were studied by changing the exit pressure ratio: the first one was a complex case with a strong shock and a separated flow region; the second was a weak shock case with attached flow throughout the entire channel. In this paper, we have focused on four sources of uncertainty: (1) discretization error, (2) error in geometry representation, (3) turbulence model, and (4) change in the downstream boundary condition.

Overall, this paper demonstrated that for the simulation of attached flows, informed CFD users can obtain reasonably accurate results, whereas they are more likely to get large errors for the cases that have strong shocks with substantial separation.

We have quantified the relative contribution and the importance of each source of uncertainty and shown the level of scatter in results that a well informed CFD user may

obtain in a typical design activity. In nozzle efficiency results, the range of variation for the strong shock case was much larger than that observed in the weak shock case. The discretization errors were up to $6 \%$ and the relative uncertainty originating from the selection of different turbulence models was as large as $9 \%$ for the strong shock case. For the weak shock case, nozzle efficiency values were more sensitive to the exit boundary 
conditions and associated error magnitudes were larger than those from other sources.

The results illustrated the interaction of different sources of uncertainty and showed that the magnitudes of numerical errors were influenced by the physical (turbulence) models used.

The results obtained in this study demonstrated that grid convergence is not achieved with grid levels that have moderate mesh sizes and showed that highly refined grids are required to obtain solutions with an acceptable level of accuracy in design problems that involve simulations of complex flow fields. The numerical noise in nozzle efficiency results observed at coarser grid levels due to the lack of grid convergence showed another impact of CFD uncertainties in design, since many gradient based optimization methods may fail to converge when the computed data has significant numerical noise.

\section{ACKNOWLEDGEMENTS}

This research was supported by the National Science Foundation grant DMI-9979711 and Air Force Office of Scientific Research grant F49620-02-1-0090. We would like to thank Dr. Rimon Arieli (Rafael, Ltd. and Technion, Israel), Dr. Christopher J. Roy (Sandia National Labs.), and Dr. Reece Neel (Aerosoft, Inc.) for their helpful comments and suggestions in the performance of this research. 


\section{Appendix A. Approximation of the discretization error by Richardson extrapolation}

A detailed description of the traditional grid convergence analysis methods, which include

the formulations given below, are presented in Roy [24]. We can write equation 2 for the nozzle efficiency results at three grid levels, grid g4, g3, and g2 as

$$
\begin{aligned}
& \left(n_{\text {eff }}\right)_{4}=\left(n_{\text {eff }}\right)_{\text {exact }}+\alpha h_{4}^{p}+O\left(h_{4}^{p+1}\right), \\
& \left(n_{\text {eff }}\right)_{3}=\left(n_{\text {eff }}\right)_{\text {exact }}+\alpha h_{3}^{p}+O\left(h_{3}^{p+1}\right), \\
& \left(n_{\text {eff }}\right)_{2}=\left(n_{\text {eff }}\right)_{\text {exact }}+\alpha h_{2}^{p}+O\left(h_{2}^{p+1}\right),
\end{aligned}
$$

where $h_{k}$ is a measure of grid spacing at grid level $k$. Since coarser grids were obtained from the finest grid level by grid halving, we have a constant grid refinement factor

$$
r=\frac{h_{1}}{h_{2}}=\frac{h_{2}}{h_{3}}=\frac{h_{3}}{h_{4}}=2.0
$$

By using equations 4 and 5 ,

$$
r^{\tilde{p}}+1=\frac{\varepsilon_{34}+\varepsilon_{23}}{\varepsilon_{34}}
$$

can be determined. Here $\tilde{p}$ is the observed order of the spatial accuracy calculated using grid levels g2, g3, and g4. The terms $\varepsilon_{23}$ and $\varepsilon_{34}$ are defined as

$$
\begin{gathered}
\varepsilon_{23}=\left(n_{e f f}\right)_{2}-\left(n_{e f f}\right)_{3}, \\
\varepsilon_{34}=\left(n_{e f f}\right)_{3}-\left(n_{e f f}\right)_{4} .
\end{gathered}
$$


From equation 6 , the observed order of spatial accuracy can be obtained as

$$
\tilde{p}=\frac{\ln \left(\varepsilon_{23} / \varepsilon_{34}\right)}{\ln (r)}
$$

By using the $n_{\text {eff }}$ results obtained at grid levels g3 and g4, we can approximate the $\left(n_{\text {eff }}\right)_{\text {exact }}$ as

$$
\left(\tilde{n}_{e f f}\right)_{\text {exact }}=\left(n_{e f f}\right)_{4}-\frac{\varepsilon_{34}}{r^{\tilde{p}}-1} .
$$

Here $\left(\tilde{n}_{\text {eff }}\right)_{\text {exact }}$ will generally be $(\tilde{p}+1)$ order accurate. Note that formulations above are derived based on the assumption that the discrete solutions obtained from three grid levels converge monotonically as the mesh size is refined. In case of non-monotonic convergence, different methods should be used. Roy [24] presented a grid convergence analysis method and an error estimation technique for mixed-order numerical schemes which exhibit non-monotonic convergence.

The values of both $\left(\tilde{n}_{\text {eff }}\right)_{\text {exact }}$ and $\tilde{p}$ depend on the grid levels used in their calculations. In Table 6, discretization error for the Sp-Al, Min-Mod, strong shock case is presented at each grid level, including g5. The first row of this table gives the $\tilde{p}$ value calculated with the results of grids g2, g3, and g4, and the $\left(\tilde{n}_{e f f}\right)_{\text {exact }}$ value obtained by using the results of grids g3 and g4. In the second row, the $\tilde{p}$ value is calculated by using the grid levels g3, g4, and g5, and the $\left(\tilde{n}_{\text {eff }}\right)_{\text {exact }}$ value is estimated by using the results from grid levels $\mathrm{g} 4$ and $\mathrm{g} 5$. The difference in $\tilde{p}$ is significant between each case. 


\section{References}

[1] Hosder, S., Watson, L. T., Grossman, B., Mason, W. H., Kim, H., Haftka, R. T., and Cox, S. E. "Polynomial Response Surface Approximations for the Multidisciplinary Design Optimization of a High Speed Civil Transport". Optimization and Engineering, 2(4):431-452, 2002.

[2] Jameson A. and Martinelli L. "Mesh Refinement and Modeling Errors in Flow Simulation". AIAA Journal, 36(5):676-686, May 1998.

[3] Levy, D. W., Zickuhr, T., Vassberg, J., Agrawal, S., Wahls, R. A., Pirzadeh, S., and Hemsch, M. J. "Summary of Data from the First AIAA CFD Drag Prediction Workshop", AIAA Paper 2002-0841, January 2002.

[4] Hemsch, M. J. "Statistical Analysis of CFD Solutions from the Drag Prediction Workshop", AIAA Paper 2002-0842, January 2002.

[5] Hemsch, M. J. and Morrison, J. H. "Statistical Analysis of CFD Solutions from $2^{\text {nd }}$ Drag Prediction Workshop", AIAA Paper 2004-0556, January 2004.

[6] Neel, R. E., Godfrey, A. G., and Slack, D. C. "Turbulence Model Validation in GASP Version 4", AIAA Paper 2003-3740, June 2003.

[7] Brown, J. L. "Turbulence Model Validation for Hypersonic Flows", AIAA Paper 2002-3308, June 2002.

[8] GASP User Manual. AeroSoft, Inc., Blacksburg, Virginia, 1997. 
[9] Oberkampf, W. L. and Blottner, F. G. "Issues in Computational Fluid Dynamics Code Verification and Validation". AIAA Journal, 36(5):687-695, May 1998.

[10] Roache, P. J. "Quantification of Uncertainty in Computational Fluid Dynamics". Annual Review of Fluid Mechanics, 29:123-160, 1997.

[11] Roache, P. J. Verification and Validation in Computational Science and Engineering. Hermosa Publishers, Albuquerque, New Mexico, 1998.

[12] Pelletier, D., Turgeon, E., Lacasse, D., and Borggaard, J. "Adaptivity, Sensitivity, and Uncertainty: Towards Standards in CFD", AIAA Paper 2001-0192, January 2001.

[13] DeLaurentis D. A. and Mavris, D. N. "Uncertainty Modeling and Management in Multidisciplinary Analysis and Synthesis", AIAA Paper 2000-0422, January 2000.

[14] "Guide for the Verification and Validation of Computational Fluid Dynamics Simulations". AIAA Standard G-07\%-1998, January 1998.

[15] Oberkampf, W. L. and Trucano, T. G. "Validation Methodology in Computational Fluid Dynamics", AIAA Paper 2000-2549, June 2000.

[16] Roache, P. J. "Error Bars for CFD", AIAA Paper 2003-0408, January 2003.

[17] Bush, R. H., Power, G. D., and Towne, C. E. "WIND: The Production Flow Solver of the NPARC Alliance", AIAA Paper 1998-0935, January 1998. 
[18] Bogar, T. J., Sajben, M., and Kroutil, J. C. "Characteristic Frequencies of Transonic Diffuser Flow Oscillations". AIAA Journal, 21(9):1232-1240, September 1983.

[19] Spalart, P. R. and Allmaras, S. R. "A One-Equation Turbulence Model for Aerodynamic Flows", AIAA Paper 1992-0439, January 1992.

[20] Wilcox, D. C. Turbulence Modeling for CFD, $2^{\text {nd }}$ Edition. DCW Industries, La Canada, California, 1998.

[21] Hosder, S., Grossman, B., Haftka, R. T., Mason, W. H., and Watson, L. T. "Remarks on CFD Simulation Uncertainties". MAD Center Report 2003-0201, (http://www.aoe.vt.edu/ mason/Mason_f/MAD030201.pdf), VPI\&SU, AOE Dept., Blacksburg, VA, February 2003.

[22] Vaidyanathan, R., Shyy, W., Garbey, M., and Haftka, R. T. "CFD Code Verification Using Least Square Extrapolation Method", AIAA Paper 2004-0739, January 2004.

[23] Carpenter, M. H. and Casper, J. H. "Accuracy of Shock Capturing in Two Spatial Dimensions". AIAA Journal, 37(9):1072-1079, September 1999.

[24] Roy, C. J. "Grid Convergence Error Analysis For Mixed-Order Numerical Schemes", AIAA Paper 2001-2006, June 2001. 


\section{List of Tables}

1 Different versions of the transonic diffuser geometry and exit pressure ratios $\left(P_{e} / P_{0 i}\right)$ used in the computations. $\ldots \ldots \ldots \ldots \ldots$

2 Mesh size nomenclature for the transonic diffuser case. In the simulations, five different grids were used for the original geometry: Grid 1 (g1), Grid 2 (g2), Grid 3 (g3), Grid 4 (g4), and Grid 5 (g5). The finest mesh is Grid 5 and the other grids are obtained by reducing the number of divisions by a factor of 2 in both $x$ - and $y$-directions at each consecutive level (grid halving). Grid 5 is used only for the case with the Sp-Al turbulence model, Min-Mod limiter, and $P_{e} / P_{0 i}=0.72$. Four grid levels were used for the extended geometry: Grid $1_{\text {ext }}\left(\mathrm{g} 1_{\text {ext }}\right)$, Grid $2_{e x t},\left(\mathrm{~g} 2_{\text {ext }}\right)$, Grid $3_{\text {ext }}\left(\mathrm{g} 3_{\text {ext }}\right)$, and Grid $4_{e x t}\left(g 4_{e x t}\right)$. The grids for the extended geometry and the grids generated for the original geometry are essentially the same between the inlet station and $x / h_{t}=8.65$. For the modified-wall geometry, three grid levels were used: Grid $1_{m w}\left(\mathrm{~g} 1_{m w}\right)$, Grid $2_{m w}\left(\mathrm{~g} 2_{m w}\right)$, and Grid $3_{m w}\left(\mathrm{~g} 3_{m w}\right)$. All the grids have the same mesh distribution in the $y$-direction. . . . . . 
3 Discretization error results of the transonic diffuser case obtained with the original geometry. The cases presented in this table exhibit monotonic convergence with the refinement of the mesh size. The observed order of accuracy $\tilde{p}$, which is also used in the $\left(\tilde{n}_{\text {eff }}\right)_{\text {exact }}$ calculations, has been calculated by using the $n_{\text {eff }}$ values from grid levels g2, g3, and g4. The approximate value of $\left(n_{e f f}\right)_{\text {exact }}$ has been calculated by using the $n_{\text {eff }}$ values obtained at grid levels g3 and g4. . . . . . . . . . . . 38

4 Nozzle efficiency values obtained with different grid levels, limiters, turbulence models, geometries, and boundary conditions. . . . . . . . . . . . 39

5 Main observations on the uncertainty in nozzle efficiencies . . . . . . . 40

6 Discretization errors calculated using the results of different grid levels with the original geometry, Sp-Al turbulence model, and the Min-Mod limiter. . . . . . . . . . . . . . . . . . . 41 


\section{List of Figures}

1 Original geometry, Grid 2 (top), and extended geometry, Grid $2_{\text {ext }}$ (bottom), used in the transonic diffuser computations. The flow is from left to right, in the positive $x$-direction. The $y$-direction is normal to the bottom wall. All dimensions are scaled by the throat height, $h_{t}$. The throat section, which is the minimum cross-sectional area of the channel, is located at $x / h_{t}=0.0$. Both geometries have the inlet stations located at $x / h_{t}=-4.04 \ldots \ldots \ldots \ldots \ldots$

2 Velocity contours, streamlines, and the top wall pressure distributions $\left(P / P_{0 i}\right)$ of the weak and the strong shock cases. The CFD results are shown for the original geometry and obtained with the Sp-Al turbulence model and the Min-Mod limiter at grid level 2. . . . . . . . . . . .

3 Nozzle efficiencies obtained with different grid levels, turbulence models, limiters, geometries, and boundary conditions for the strong shock case (A) and the weak shock case $(\mathrm{B}) . \ldots \ldots \ldots$

4 Discretization errors and exact values of nozzle efficiencies approximated with Richardson extrapolation for different turbulence models and limiters at each grid level for the weak shock case. The discretization errors are represented with one-sided error bars. . . . . . . . . . . . . . 
5 Discretization errors and exact values of nozzle efficiencies approximated with Richardson extrapolation for different turbulence models and limiters at each grid level for the strong shock case. Only the cases that have monotonic grid convergence are shown, and the discretization errors are represented with one-sided error bars. . . . . . . . . . . . . . . 46

6 Mach number values at the upstream of the shock $\left(x / h_{t}=-1.5\right)$, and downstream of the shock $\left(x / h_{t}=8.65\right.$, the exit plane) for different grids obtained with the original geometry, $\mathrm{Sp}-\mathrm{Al}$ and $k-\omega$ turbulence models, Min-Mod and Van Albada limiters. The values of $y / h_{t}$ correspond to the mid points of the local channel heights. . . . . . . . . . . . . . .

$7 \quad$ Nozzle efficiency vs. exit pressure ratio for different grids obtained with the original geometry, Sp-Al and $k$ - $\omega$ turbulence models, and the Min-Mod limiter. Note that grid 4 results are presented only for $P_{e} / P_{0 i}=0.72$ and 0.82, and grid 5 result is given only for $P_{e} / P_{0 i}=0.72$ and the Sp-Al model. 48

8 Error distribution in $y / h_{t}$ for the upper wall of the modified-wall diffuser geometry at the data points measured in the experiments. The maximum error is approximately $7 \%$ and observed upstream of the throat, at $x / h_{t}=$ -1.95. Starting from $x / h_{t}=1.2$, the error is approximately constant with an average value of $0.9 \% \ldots \ldots \ldots \ldots$ 
9 Upper wall contours of the original and the modified-wall diffuser geometry in the vicinity of the throat location. . . . . . . . . . . . 50

10 Top wall pressure distributions obtained with the original and the modifiedwall geometry for the strong shock case (the results of the Sp-Al model, Min-Mod limiter, and Grids g2 and g2 $2_{m w}$ are shown). . . . . . . . . . . 51

11 Top wall pressure distributions obtained with the original and the modifiedwall geometry for the weak shock case (the results of the Sp-Al model, Min-Mod limiter, and Grids g2 and $\mathrm{g} 2_{m w}$ are shown). . . . . . . . . . . 52

12 Streamline patterns of the separated flow region obtained with different versions of the diffuser geometry and exit pressure ratios for the strong

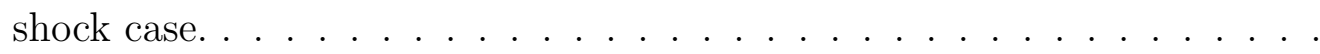

13 Comparison of the separation bubbles obtained with different versions of the diffuser geometry and exit pressure ratios for the strong shock case.

14 Top wall pressure distributions obtained with different versions of the diffuser geometry and exit pressure ratios for the strong shock case (the results of the Sp-Al model, Van Albada limiter, and Grids g3 and g3 $3_{\text {ext }}$ are shown). . . . . . . . . . . . . . . . 
Table 1: Different versions of the transonic diffuser geometry and exit pressure ratios $\left(P_{e} / P_{0 i}\right)$ used in the computations.

\begin{tabular}{ccc} 
Geometry & $\begin{array}{c}x / h_{t} \text { at the } \\
\text { exit station }\end{array}$ & $P_{e} / P_{0 i}$ \\
\hline \hline original & 8.65 & 0.72 and 0.82 \\
\hline modified-wall & 8.65 & 0.72 and 0.82 \\
\hline extended & 14.44 & $0.72,0.7468$ \\
& & 0.82, and 0.8368 \\
\hline \hline
\end{tabular}


Table 2: Mesh size nomenclature for the transonic diffuser case. In the simulations, five different grids were used for the original geometry: Grid 1 (g1), Grid 2 (g2), Grid 3 (g3), Grid 4 (g4), and Grid 5 (g5). The finest mesh is Grid 5 and the other grids are obtained by reducing the number of divisions by a factor of 2 in both $x$ - and $y$-directions at each consecutive level (grid halving). Grid 5 is used only for the case with the Sp-Al turbulence model, Min-Mod limiter, and $P_{e} / P_{0 i}=0.72$. Four grid levels were used for the extended geometry: Grid $1_{\text {ext }}\left(\mathrm{g} 1_{\text {ext }}\right)$, Grid $2_{e x t}$, $\left(\mathrm{g} 2_{e x t}\right)$, Grid $3_{e x t}\left(\mathrm{~g} 3_{e x t}\right)$, and Grid $4_{e x t}\left(g 4_{e x t}\right)$. The grids for the extended geometry and the grids generated for the original geometry are essentially the same between the inlet station and $x / h_{t}=8.65$. For the modified-wall geometry, three grid levels were used: Grid $1_{m w}$ $\left(\mathrm{g} 1_{m w}\right)$, Grid $2_{m w}\left(\mathrm{~g} 2_{m w}\right)$, and Grid $3_{m w}\left(\mathrm{~g} 3_{m w}\right)$. All the grids have the same mesh distribution in the $y$-direction.

\begin{tabular}{ccc} 
Grid & $\begin{array}{c}x / h_{t} \text { at the } \\
\text { exit station }\end{array}$ & mesh size \\
\hline \hline $\mathrm{g} 1$ & 8.65 & $41 \times 26 \times 2$ \\
\hline $\mathrm{g} 2$ & 8.65 & $81 \times 51 \times 2$ \\
\hline $\mathrm{g} 3$ & 8.65 & $161 \times 101 \times 2$ \\
\hline $\mathrm{g} 4$ & 8.65 & $321 \times 201 \times 2$ \\
\hline $\mathrm{g} 5$ & 8.65 & $641 \times 401 \times 2$ \\
\hline $\mathrm{g} 1_{\text {ext }}$ & 14.44 & $46 \times 26 \times 2$ \\
\hline $\mathrm{g} 2_{e x t}$ & 14.44 & $91 \times 51 \times 2$ \\
\hline $\mathrm{g} 3_{\text {ext }}$ & 14.44 & $181 \times 101 \times 2$ \\
\hline $\mathrm{g} 4_{e x t}$ & 14.44 & $361 \times 201 \times 2$ \\
\hline $\mathrm{g} 1_{m w}$ & 8.65 & $41 \times 26 \times 2$ \\
\hline $\mathrm{g} 2_{m w}$ & 8.65 & $81 \times 51 \times 2$ \\
\hline $\mathrm{g} 3_{m w}$ & 8.65 & $161 \times 101 \times 2$ \\
\hline \hline
\end{tabular}


Table 3: Discretization error results of the transonic diffuser case obtained with the original geometry. The cases presented in this table exhibit monotonic convergence with the refinement of the mesh size. The observed order of accuracy $\tilde{p}$, which is also used in the $\left(\tilde{n}_{\text {eff }}\right)_{\text {exact }}$ calculations, has been calculated by using the $n_{\text {eff }}$ values from grid levels g2, g3, and g4. The approximate value of $\left(n_{\text {eff }}\right)_{\text {exact }}$ has been calculated by using the $n_{\text {eff }}$ values obtained at grid levels g3 and g4.

\begin{tabular}{|c|c|c|c|c|c|c|}
\hline $\begin{array}{c}\text { turbulence } \\
\text { model }\end{array}$ & limiter & $P_{e} / P_{0 i}$ & $\tilde{p}$ & $\left(\tilde{n}_{\text {eff }}\right)_{\text {exact }}$ & $\begin{array}{l}\text { grid } \\
\text { level }\end{array}$ & $\begin{array}{c}\text { discretization } \\
\text { error }(\%)\end{array}$ \\
\hline \multirow{4}{*}{$\mathrm{Sp}-\mathrm{Al}$} & \multirow{4}{*}{ Van Albada } & \multirow{4}{*}{0.72} & \multirow{4}{*}{1.528} & \multirow{4}{*}{0.71830} & g1 & 9.820 \\
\hline & & & & & g2 & 4.505 \\
\hline & & & & & g3 & 1.562 \\
\hline & & & & & g4 & 0.542 \\
\hline \multirow{4}{*}{$\mathrm{Sp}-\mathrm{Al}$} & \multirow{4}{*}{ Min-Mod } & \multirow{4}{*}{0.72} & \multirow{4}{*}{1.322} & \multirow{4}{*}{0.71590} & g1 & 14.298 \\
\hline & & & & & g2 & 6.790 \\
\hline & & & & & g3 & 2.716 \\
\hline & & & & & g4 & 1.086 \\
\hline \multirow{4}{*}{$\mathrm{Sp}-\mathrm{Al}$} & \multirow{4}{*}{ Van Albada } & \multirow{4}{*}{0.82} & \multirow{4}{*}{1.198} & \multirow{4}{*}{0.80958} & g1 & 6.761 \\
\hline & & & & & g2 & 3.507 \\
\hline & & & & & g3 & 1.528 \\
\hline & & & & & g4 & 0.666 \\
\hline \multirow{4}{*}{$\mathrm{Sp}-\mathrm{Al}$} & \multirow{4}{*}{ Min-Mod } & \multirow{4}{*}{0.82} & \multirow{4}{*}{1.578} & \multirow{4}{*}{0.81086} & g1 & 8.005 \\
\hline & & & & & g2 & 3.539 \\
\hline & & & & & g3 & 1.185 \\
\hline & & & & & g4 & 0.397 \\
\hline \multirow{4}{*}{$k-\omega$} & \multirow{4}{*}{ Van Albada } & \multirow{4}{*}{0.82} & \multirow{4}{*}{1.980} & \multirow{4}{*}{0.82962} & g1 & 3.514 \\
\hline & & & & & g2 & 1.459 \\
\hline & & & & & g3 & 0.370 \\
\hline & & & & & g4 & 0.094 \\
\hline \multirow{4}{*}{$k-\omega$} & \multirow{4}{*}{ Min-Mod } & \multirow{4}{*}{0.82} & \multirow{4}{*}{1.656} & \multirow{4}{*}{0.82889} & g1 & 4.432 \\
\hline & & & & & g2 & 1.452 \\
\hline & & & & & g3 & 0.461 \\
\hline & & & & & g4 & 0.146 \\
\hline
\end{tabular}


Table 4: Nozzle efficiency values obtained with different grid levels, limiters, turbulence models, geometries, and boundary conditions.

\begin{tabular}{|c|c|c|c|c|c|c|c|c|c|c|}
\hline \multirow{3}{*}{$\begin{array}{l}\text { turbulence } \\
\text { model }\end{array}$} & \multirow{3}{*}{ limiter } & \multirow{3}{*}{$\begin{array}{l}\text { grid } \\
\text { level }\end{array}$} & \multicolumn{4}{|c|}{ strong shock } & \multicolumn{4}{|c|}{ weak shock } \\
\hline & & & \multirow{2}{*}{$\begin{array}{l}\text { original } \\
\text { geometry }\end{array}$} & \multirow{2}{*}{$\begin{array}{l}\text { modified- } \\
\text { wall } \\
\text { geometry }\end{array}$} & \multicolumn{2}{|c|}{$\begin{array}{l}\text { extended } \\
\text { geometry }\end{array}$} & \multirow{2}{*}{$\begin{array}{l}\text { original } \\
\text { geometry }\end{array}$} & \multirow{2}{*}{$\begin{array}{l}\text { modified- } \\
\text { wall } \\
\text { geometry }\end{array}$} & \multicolumn{2}{|c|}{$\begin{array}{l}\text { extended } \\
\text { geometry }\end{array}$} \\
\hline & & & & & $\begin{array}{l}P_{e} / P_{0 i} \\
0.7468\end{array}$ & $\begin{array}{c}P_{e} / P_{0 i} \\
0.72\end{array}$ & & & $\begin{array}{c}P_{e} / P_{0 i} \\
0.8368\end{array}$ & $\begin{array}{c}P_{e} / P_{0 i} \\
0.82\end{array}$ \\
\hline \multirow{4}{*}{$k-\omega$} & \multirow{4}{*}{ Min-mod } & 1 & 0.81113 & 0.80556 & & & 0.86563 & 0.86158 & & \\
\hline & & 2 & 0.79362 & 0.79640 & & & 0.84093 & 0.83297 & & \\
\hline & & 3 & 0.78543 & 0.78886 & & & 0.83271 & 0.82249 & & \\
\hline & & 4 & 0.79007 & & & & 0.83011 & & & \\
\hline \multirow{4}{*}{$k-\omega$} & \multirow{4}{*}{ Van Albada } & 1 & 0.78820 & 0.78333 & & & 0.85879 & 0.84477 & & \\
\hline & & 2 & 0.78199 & 0.78439 & & & 0.84174 & 0.83420 & & \\
\hline & & 3 & 0.78310 & 0.78661 & & & 0.83270 & 0.82237 & & \\
\hline & & 4 & 0.78788 & & & & 0.83041 & & & \\
\hline \multirow{4}{*}{$\mathrm{Sp}-\mathrm{Al}$} & \multirow{4}{*}{ Min-mod } & 1 & 0.81827 & 0.81562 & & & 0.87577 & 0.86931 & & \\
\hline & & 2 & 0.76452 & 0.76479 & & & 0.83956 & 0.83290 & & \\
\hline & & 3 & 0.73535 & 0.73402 & & & 0.82048 & 0.81409 & & \\
\hline & & 4 & 0.72369 & & & & 0.81408 & & & \\
\hline \multirow{4}{*}{ Sp-Al } & \multirow{4}{*}{ Van Albada } & 1 & 0.78885 & 0.78647 & 0.78855 & 0.77702 & 0.86432 & 0.85336 & 0.89069 & 0.85429 \\
\hline & & 2 & 0.75067 & 0.74850 & 0.75777 & 0.75072 & 0.83797 & 0.83172 & 0.87461 & 0.82993 \\
\hline & & 3 & 0.72953 & 0.72569 & 0.74231 & 0.73526 & 0.82195 & 0.81586 & 0.86819 & 0.81664 \\
\hline & & 4 & 0.72220 & & 0.73268 & 0.72517 & 0.81497 & & 0.86464 & 0.81130 \\
\hline
\end{tabular}


Table 5: Main observations on the uncertainty in nozzle efficiencies

\section{Shock type Observations on uncertainties}

Strong shock The range of variation in nozzle efficiency results is much larger than that case observed in the weak shock case. The maximum variation is about $10 \%$ for the strong shock case, and $4 \%$ for the weak shock case, when the results of the original geometry are compared.

Magnitude of the discretization errors is larger than that of the weak shock case. The discretization errors at grid level 2 can be up to $6 \%$ for the strong shock case.

Relative uncertainty due to the selection of the turbulence model can be larger than that due to discretization errors depending upon the grid level used. This uncertainty can be as large as $9 \%$ at grid level 4 .

The contribution of the error in geometry representation to the overall uncertainty is negligible compared to the other sources of uncertainty.

Weak shock For the results obtained with the original geometry, the maximum value of case the discretization error is $3.5 \%$, whereas the maximum value of turbulence model uncertainty is about $2 \%$.

The nozzle efficiency values are more sensitive to the exit boundary conditions and associated error magnitudes can be larger than from other sources. The difference between the results from original geometry and the extended geometry can be as large as $7 \%$ when the exit pressure ratio of 0.8368 is used.

The contribution of the error in geometry representation to the overall uncertainty can be up to $1.4 \%$. 
Table 6: Discretization errors calculated using the results of different grid levels with the original geometry, Sp-Al turbulence model, and the Min-Mod limiter.

\begin{tabular}{|c|c|c|c|c|}
\hline $\begin{array}{c}\text { grid levels } \\
\text { used }\end{array}$ & $\tilde{p}$ & $\left(\tilde{n}_{e f f}\right)_{\text {exact }}$ & $\begin{array}{l}\text { grid } \\
\text { level }\end{array}$ & $\begin{array}{c}\text { error } \\
(\%)\end{array}$ \\
\hline \multirow{5}{*}{$\begin{array}{l}\text { for } \tilde{p}: \\
\text { g2, g3, and g4 } \\
\text { for }\left(\tilde{n}_{\text {eff }}\right)_{\text {exact }} \text { : } \\
\text { g3 and g4 }\end{array}$} & \multirow{5}{*}{1.322} & \multirow{5}{*}{0.71590} & g1 & 14.298 \\
\hline & & & g2 & 6.790 \\
\hline & & & g3 & 2.716 \\
\hline & & & $\mathrm{g} 4$ & 1.086 \\
\hline & & & g5 & 0.634 \\
\hline \multirow{5}{*}{$\begin{array}{l}\text { for } \tilde{p}: \\
\text { g3, g4, and g5 } \\
\text { for }\left(\tilde{n}_{\text {eff }}\right)_{\text {exact }} \text { : } \\
\text { g4 and g5 }\end{array}$} & \multirow{5}{*}{1.849} & \multirow{5}{*}{0.71921} & g1 & 13.774 \\
\hline & & & g2 & 6.300 \\
\hline & & & g3 & 2.245 \\
\hline & & & g4 & 0.623 \\
\hline & & & g5 & 0.173 \\
\hline
\end{tabular}



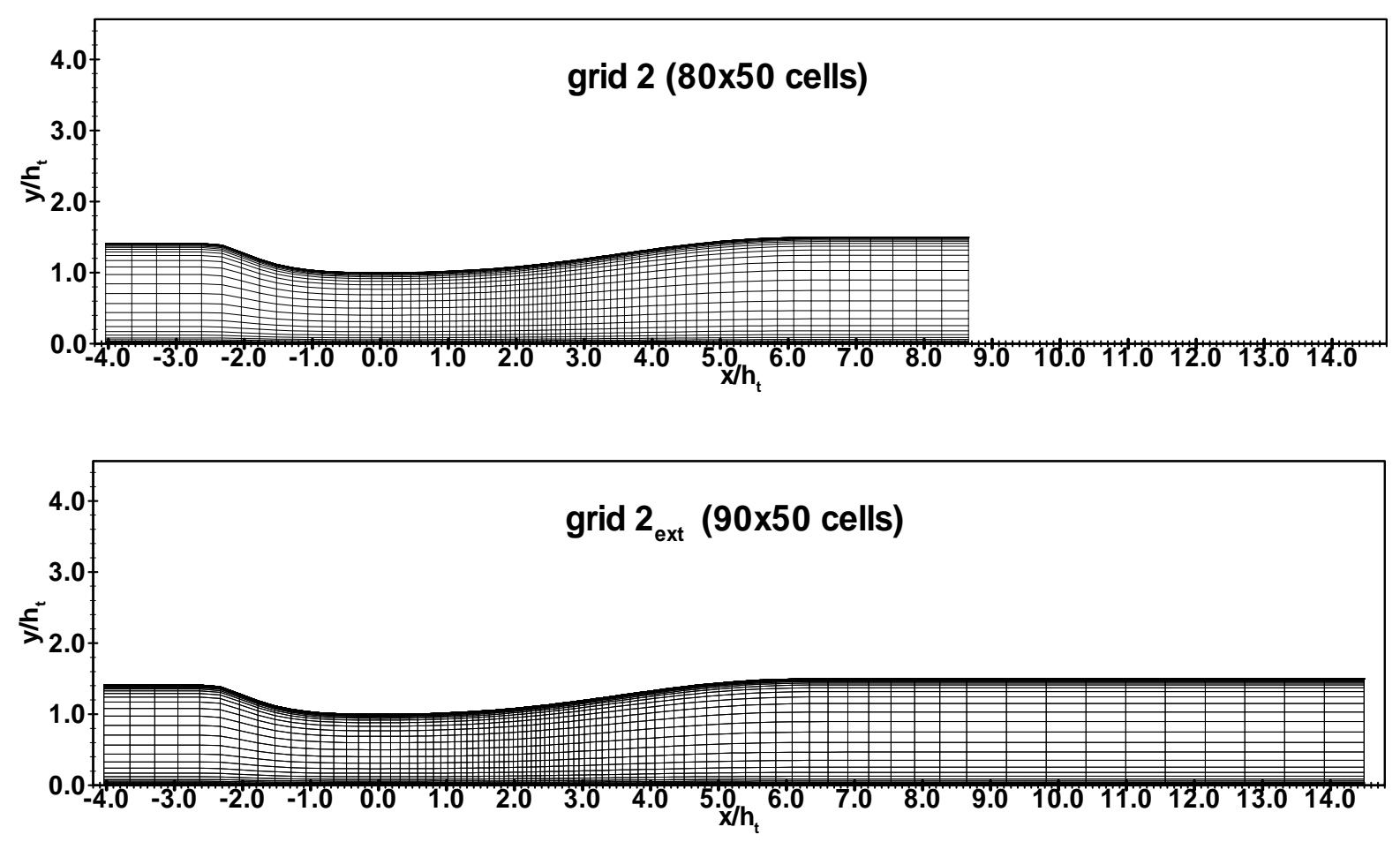

Figure 1: Original geometry, Grid 2 (top), and extended geometry, Grid $2_{\text {ext }}$ (bottom), used in the transonic diffuser computations. The flow is from left to right, in the positive $x$-direction. The $y$-direction is normal to the bottom wall. All dimensions are scaled by the throat height, $h_{t}$. The throat section, which is the minimum cross-sectional area of the channel, is located at $x / h_{t}=0.0$. Both geometries have the inlet stations located at $x / h_{t}=-4.04$. 

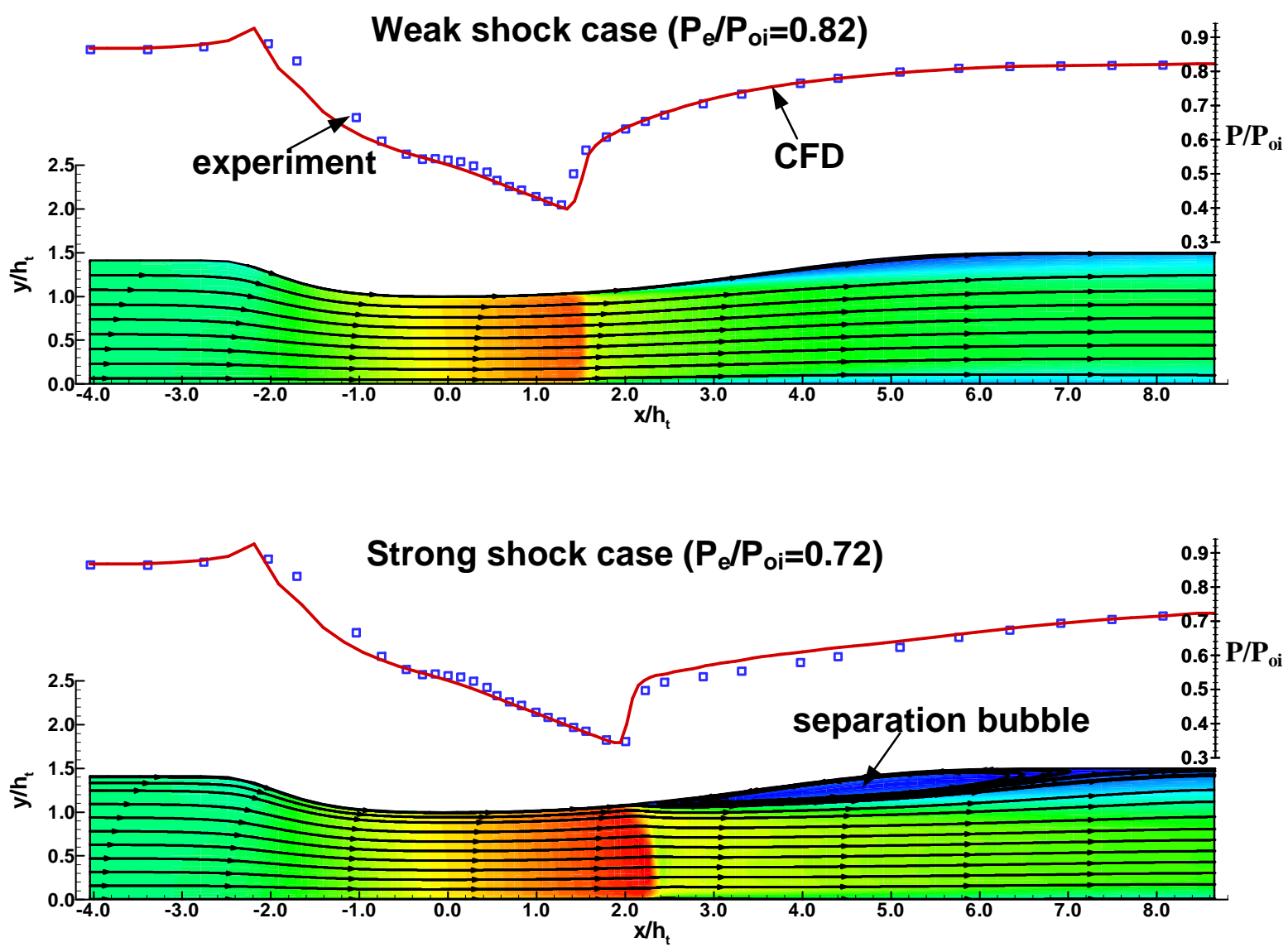

Figure 2: Velocity contours, streamlines, and the top wall pressure distributions $\left(P / P_{0 i}\right)$ of the weak and the strong shock cases. The CFD results are shown for the original geometry and obtained with the Sp-Al turbulence model and the Min-Mod limiter at grid level 2. 


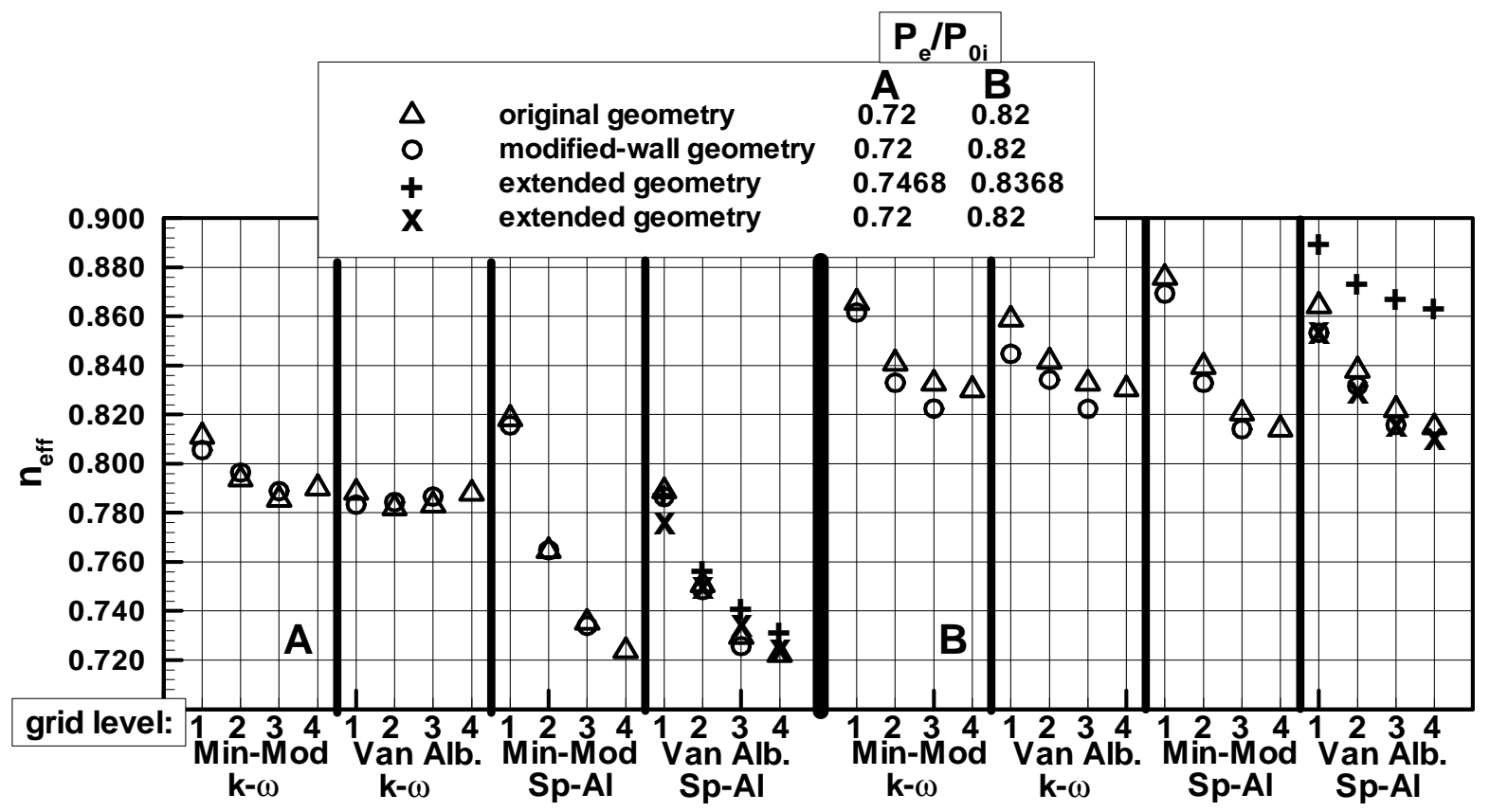

Figure 3: Nozzle efficiencies obtained with different grid levels, turbulence models, limiters, geometries, and boundary conditions for the strong shock case (A) and the weak shock case (B). 

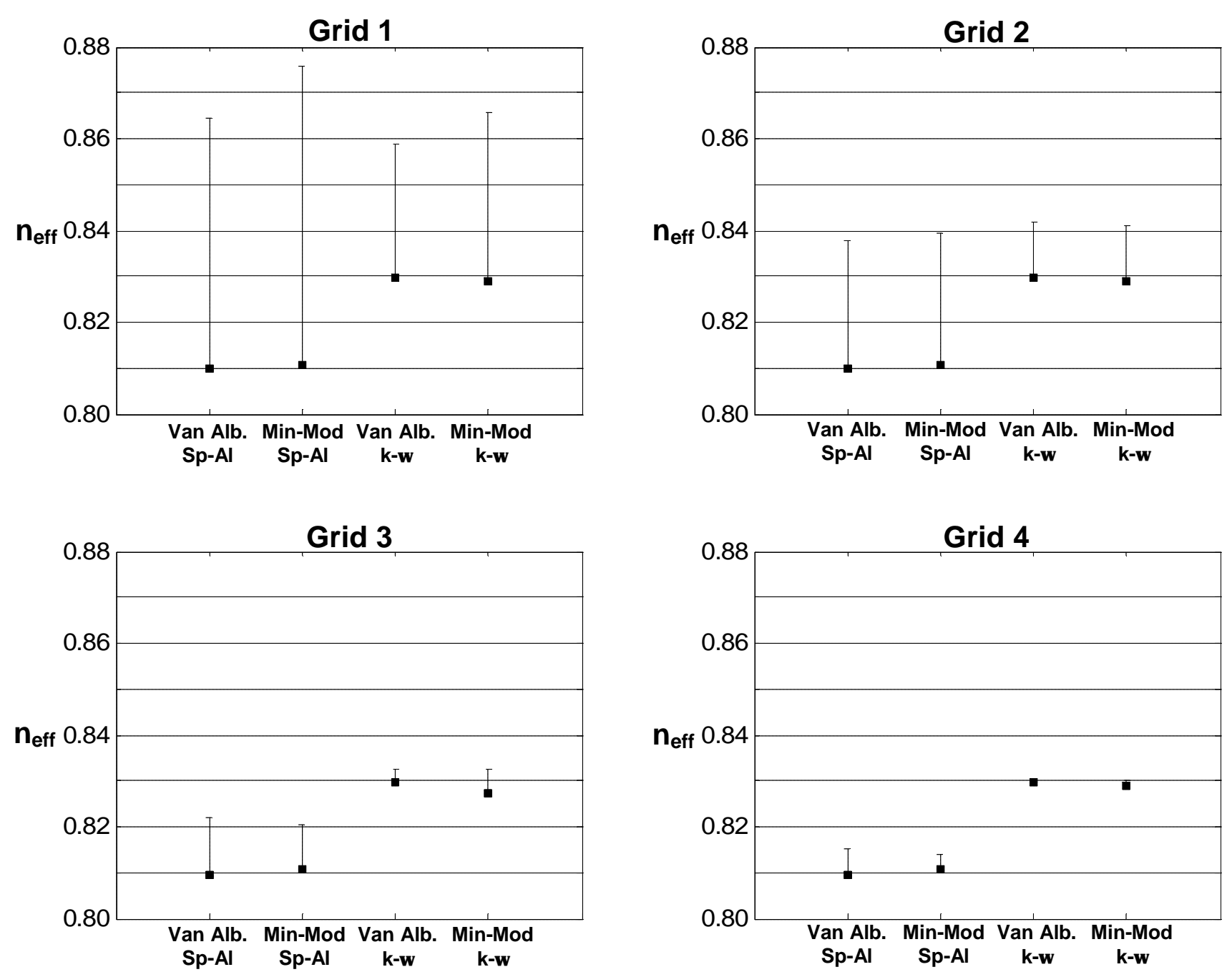

Figure 4: Discretization errors and exact values of nozzle efficiencies approximated with Richardson extrapolation for different turbulence models and limiters at each grid level for the weak shock case. The discretization errors are represented with one-sided error bars. 

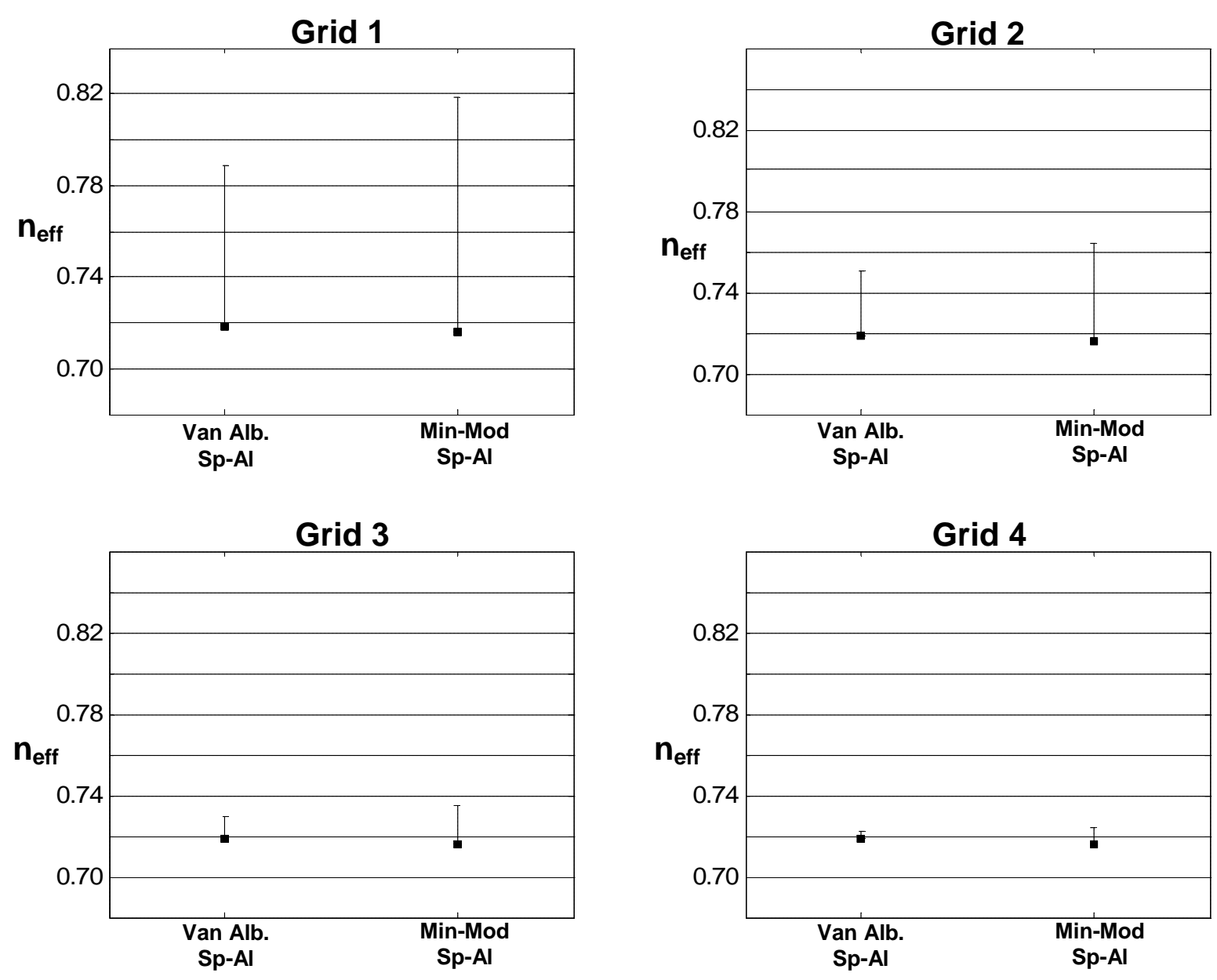

Figure 5: Discretization errors and exact values of nozzle efficiencies approximated with Richardson extrapolation for different turbulence models and limiters at each grid level for the strong shock case. Only the cases that have monotonic grid convergence are shown, and the discretization errors are represented with one-sided error bars. 


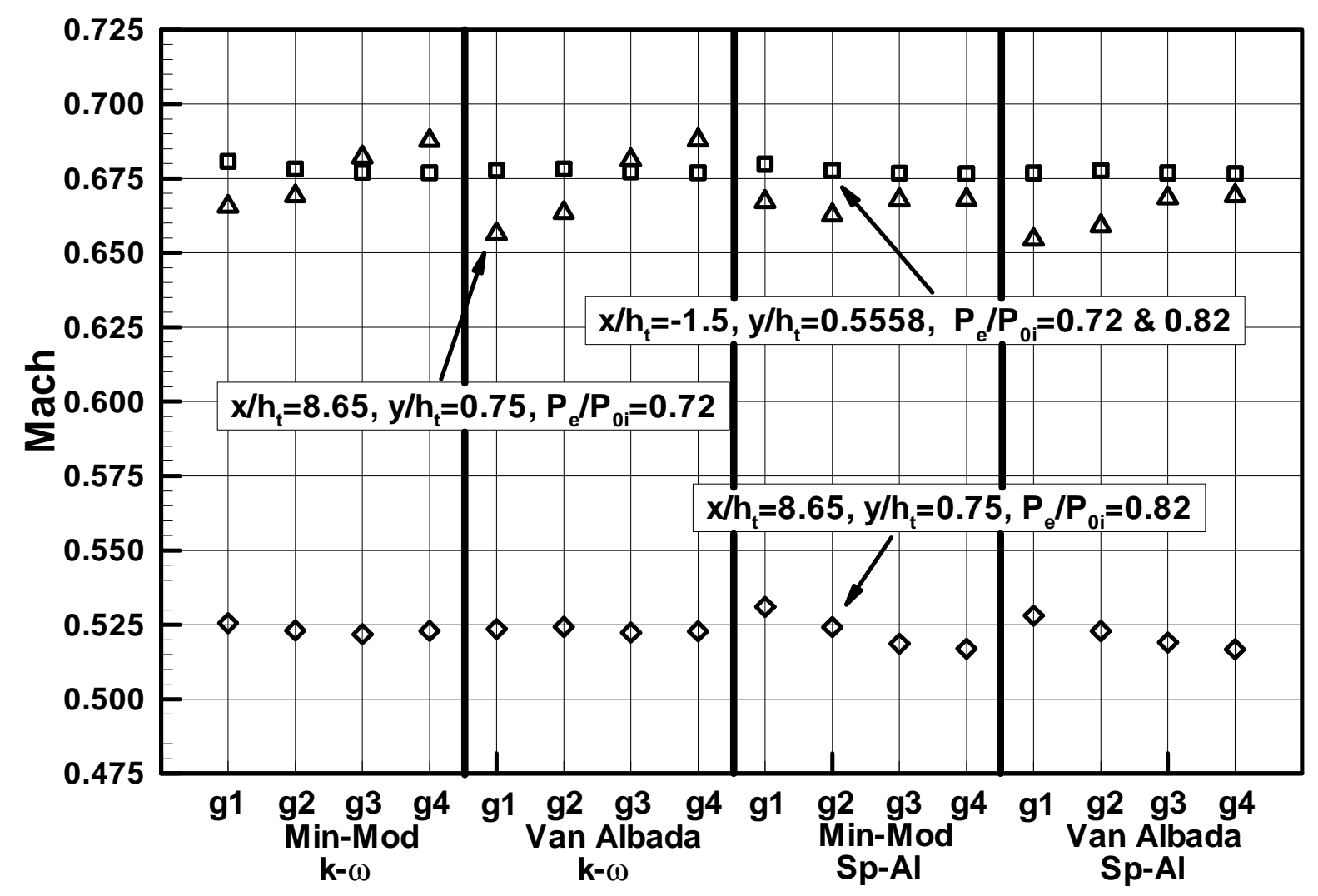

Figure 6: Mach number values at the upstream of the shock $\left(x / h_{t}=-1.5\right)$, and downstream of the shock $\left(x / h_{t}=8.65\right.$, the exit plane) for different grids obtained with the original geometry, $\mathrm{Sp}-\mathrm{Al}$ and $k-\omega$ turbulence models, Min-Mod and Van Albada limiters. The values of $y / h_{t}$ correspond to the mid points of the local channel heights. 


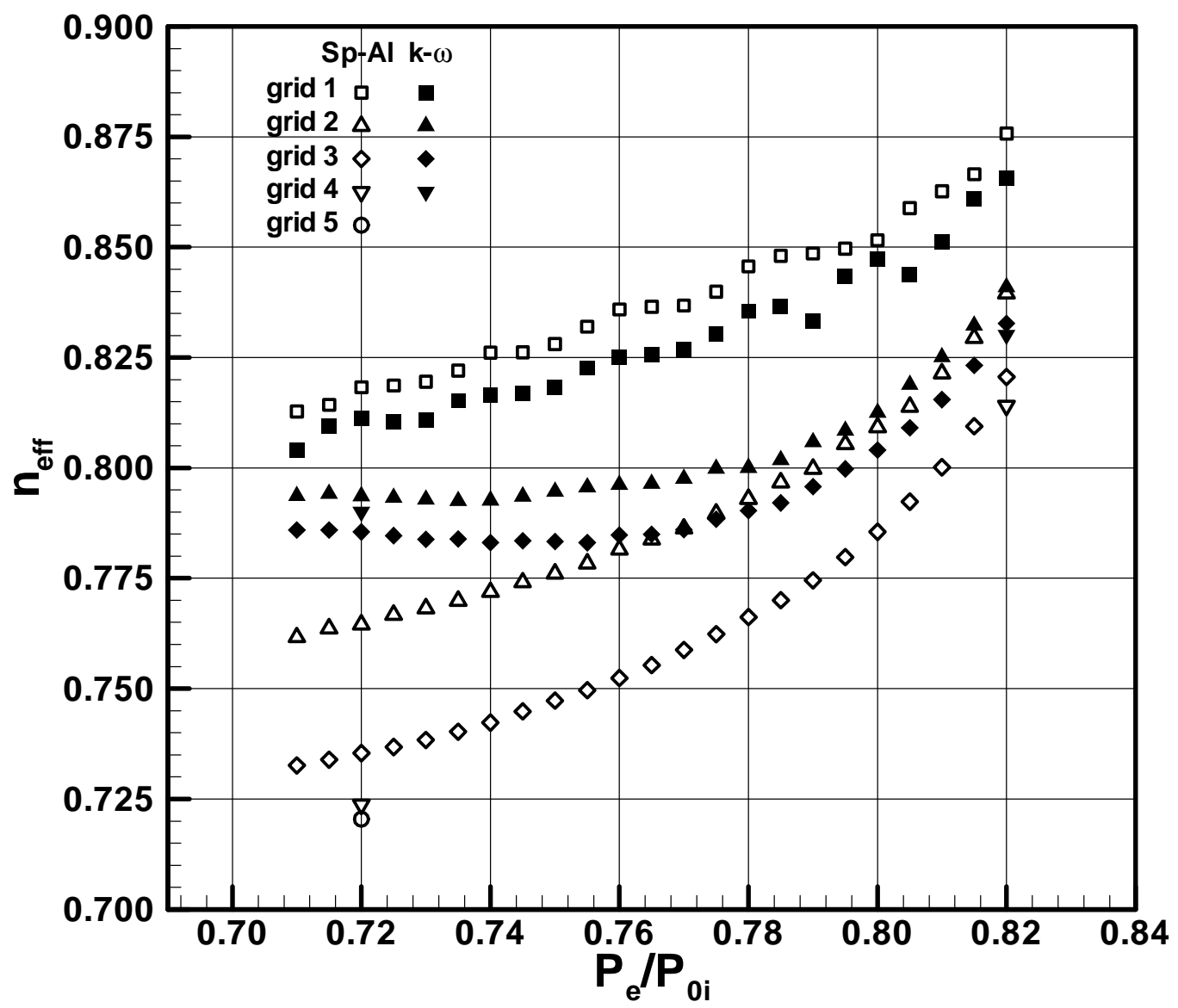

Figure 7: Nozzle efficiency vs. exit pressure ratio for different grids obtained with the original geometry, Sp-Al and $k-\omega$ turbulence models, and the Min-Mod limiter. Note that grid 4 results are presented only for $P_{e} / P_{0 i}=0.72$ and 0.82 , and grid 5 result is given only for $P_{e} / P_{0 i}=0.72$ and the $\mathrm{Sp}-\mathrm{Al}$ model. 


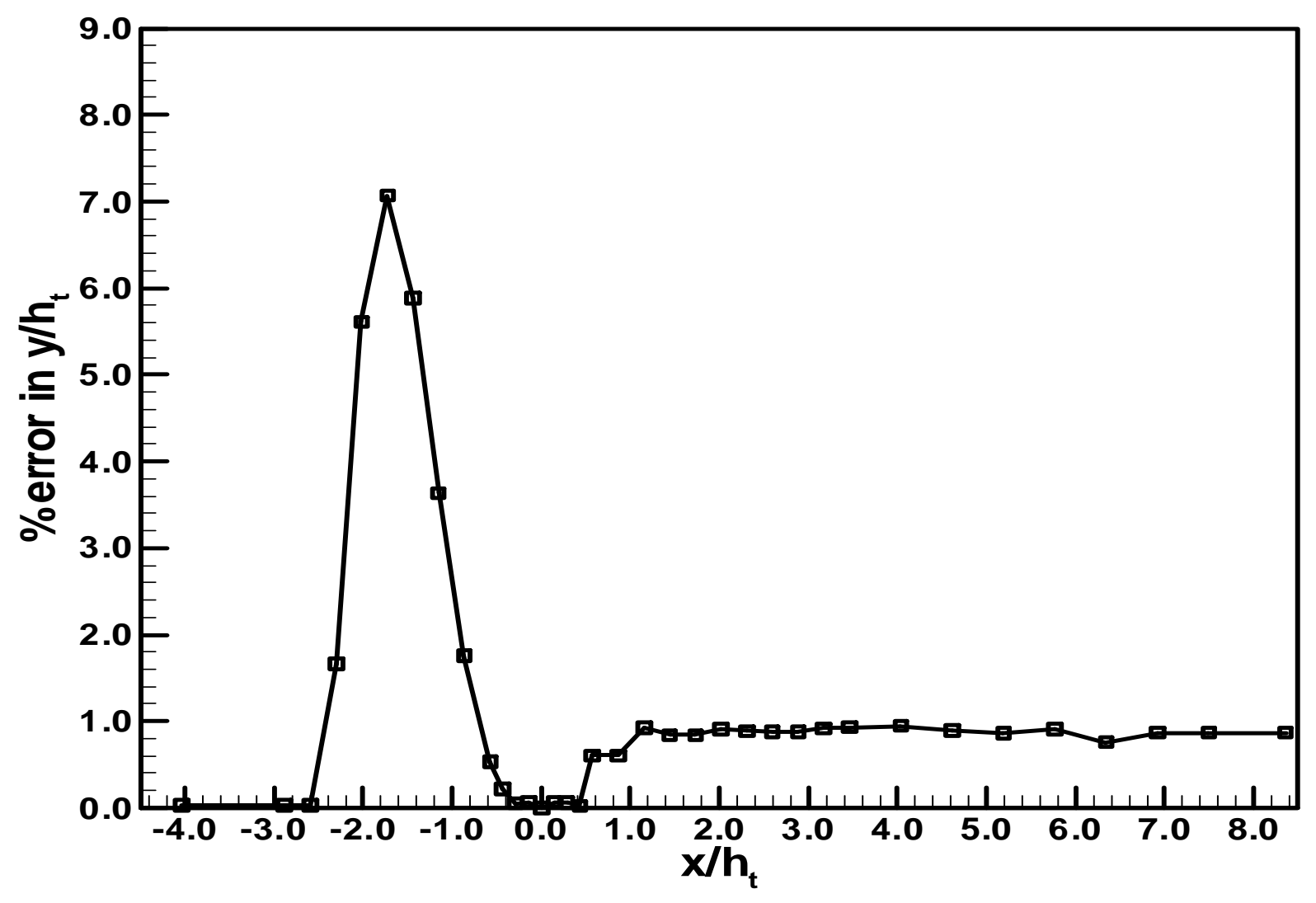

Figure 8: Error distribution in $y / h_{t}$ for the upper wall of the modified-wall diffuser geometry at the data points measured in the experiments. The maximum error is approximately $7 \%$ and observed upstream of the throat, at $x / h_{t}=-1.95$. Starting from $x / h_{t}=1.2$, the error is approximately constant with an average value of $0.9 \%$. 


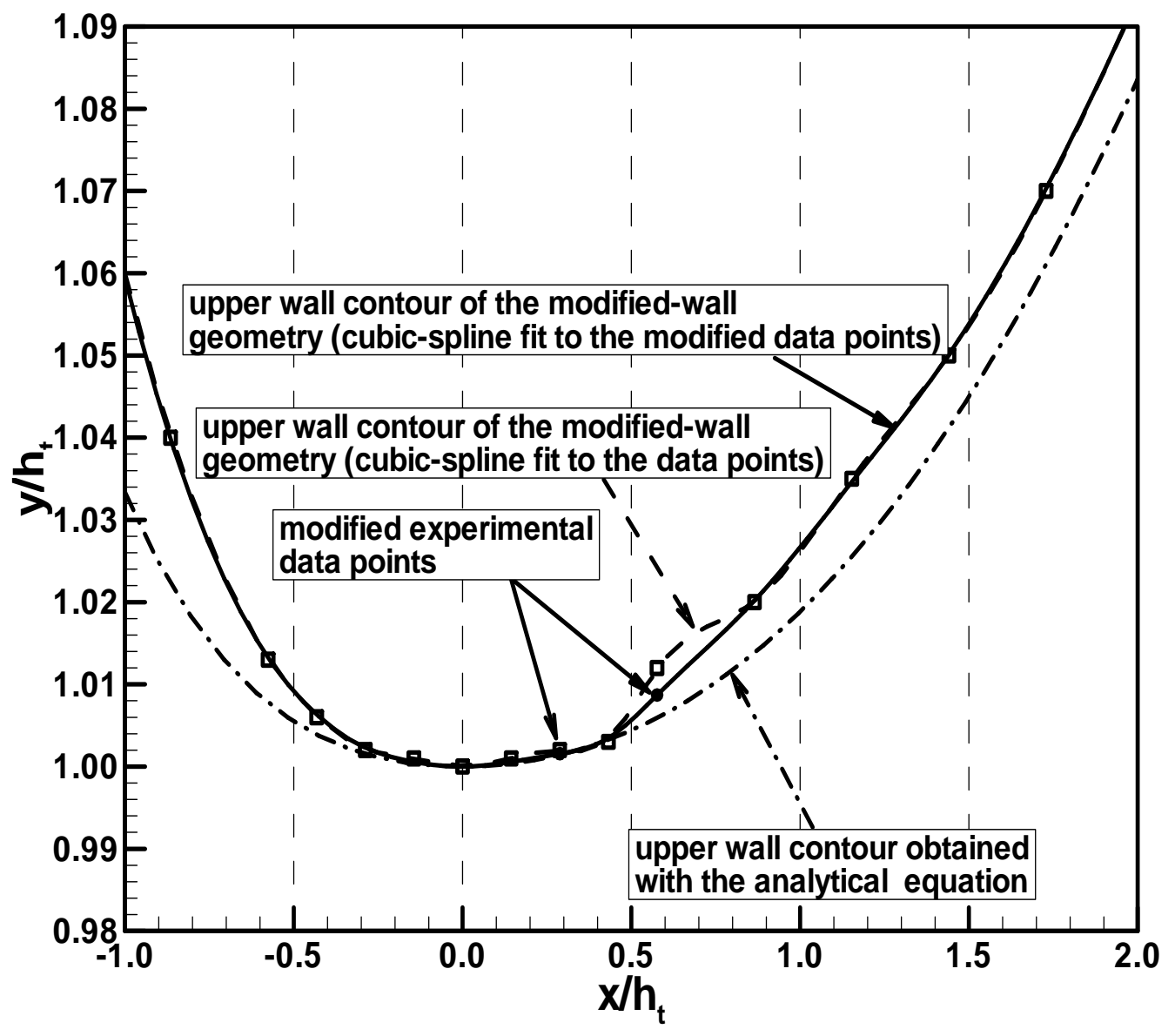

Figure 9: Upper wall contours of the original and the modified-wall diffuser geometry in the vicinity of the throat location. 


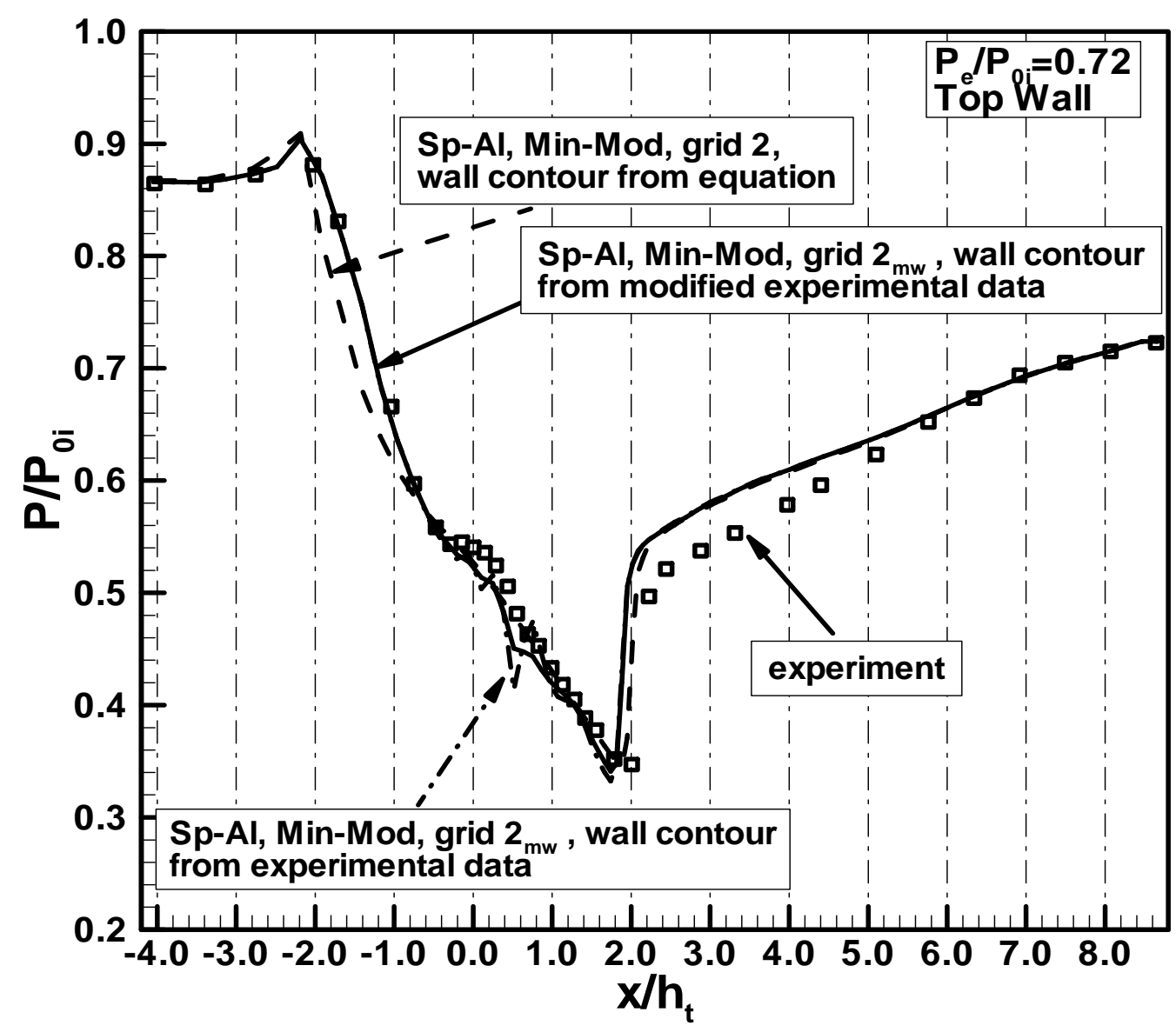

Figure 10: Top wall pressure distributions obtained with the original and the modified-wall geometry for the strong shock case (the results of the Sp-Al model, Min-Mod limiter, and Grids g2 and $\mathrm{g} 2{ }_{m w}$ are shown). 


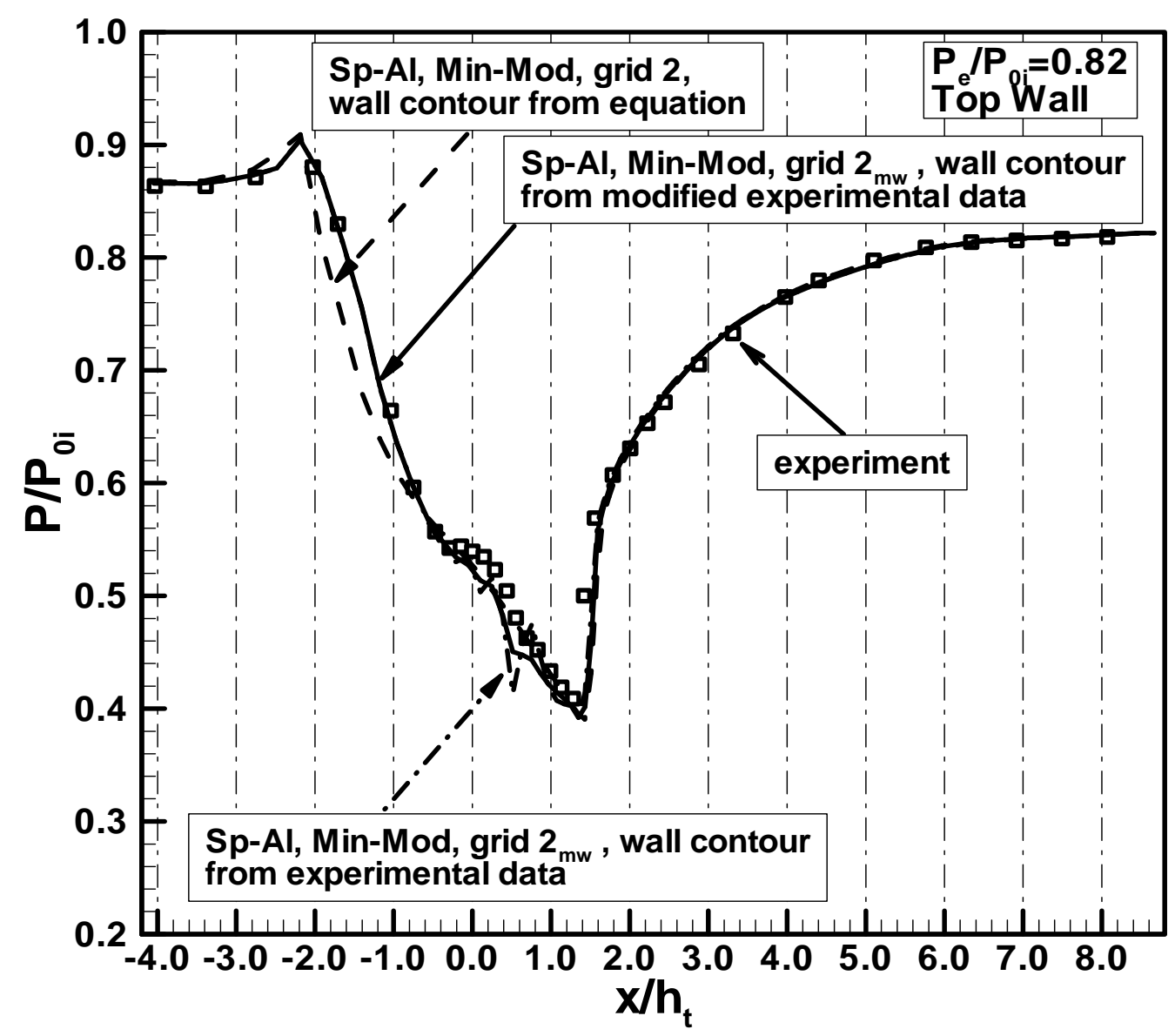

Figure 11: Top wall pressure distributions obtained with the original and the modified-wall geometry for the weak shock case (the results of the Sp-Al model, Min-Mod limiter, and Grids g2 and $\mathrm{g} 2_{m w}$ are shown). 

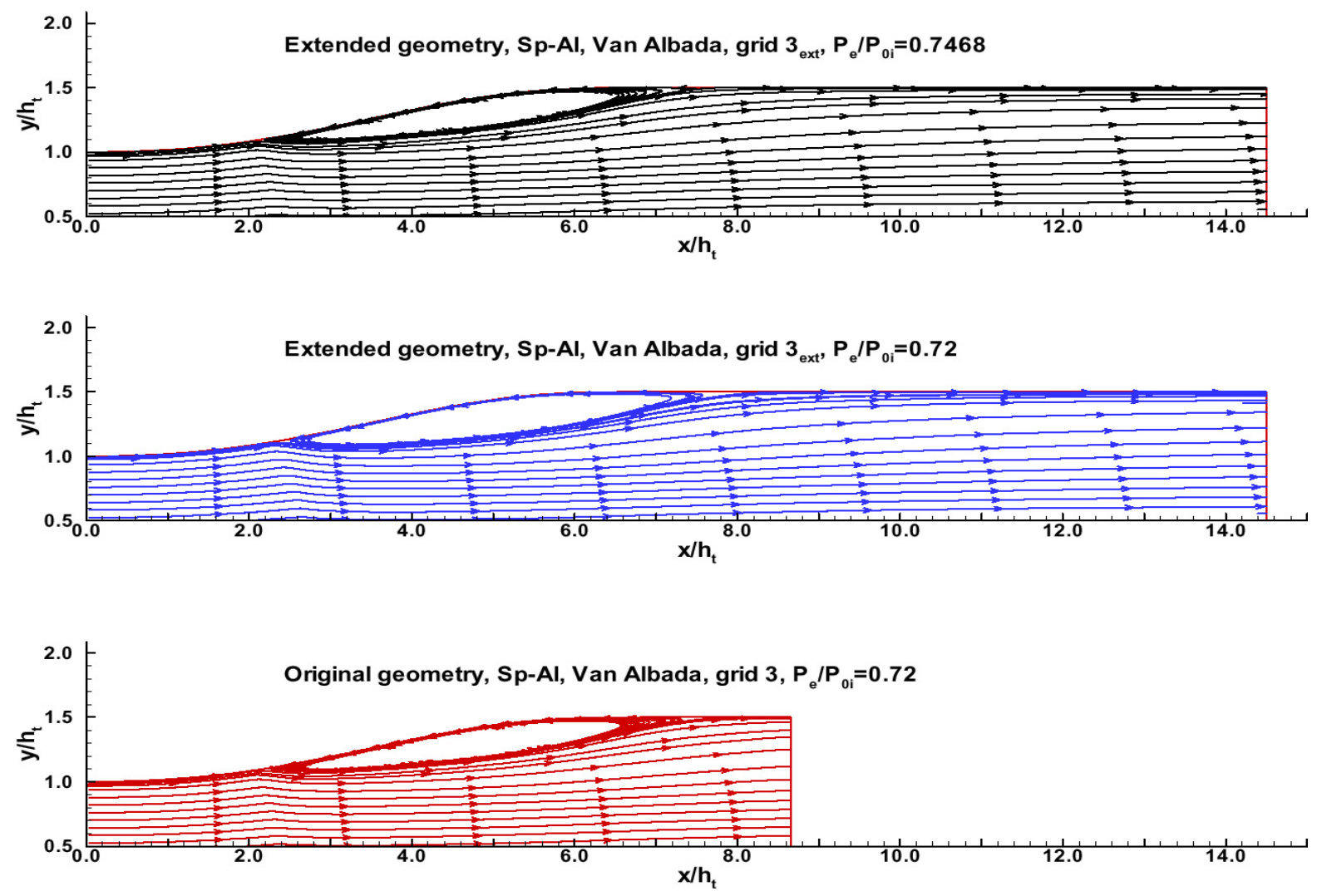

Figure 12: Streamline patterns of the separated flow region obtained with different versions of the diffuser geometry and exit pressure ratios for the strong shock case. 


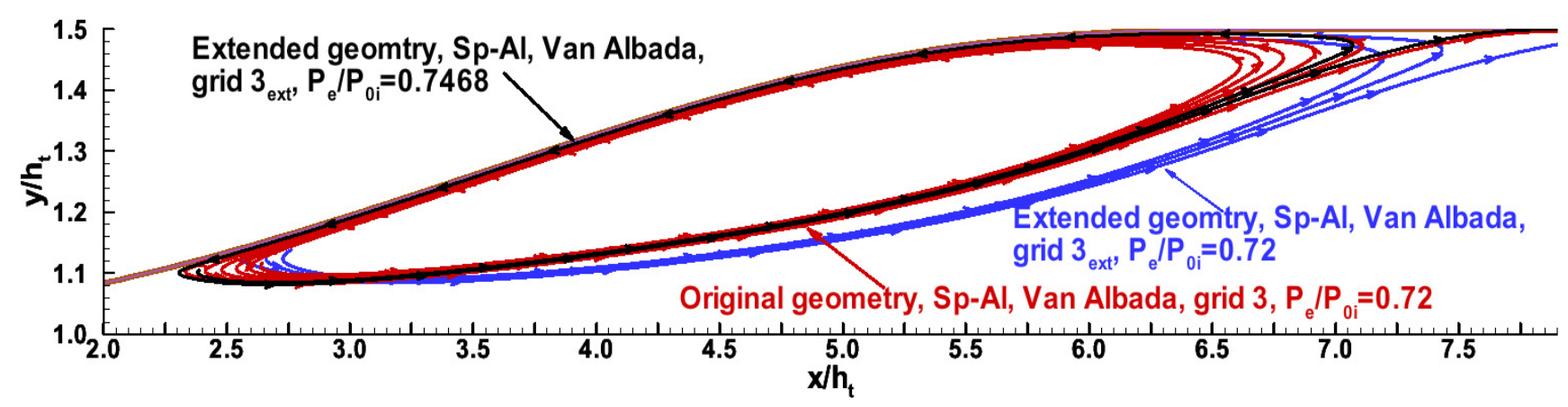

Figure 13: Comparison of the separation bubbles obtained with different versions of the diffuser geometry and exit pressure ratios for the strong shock case. 


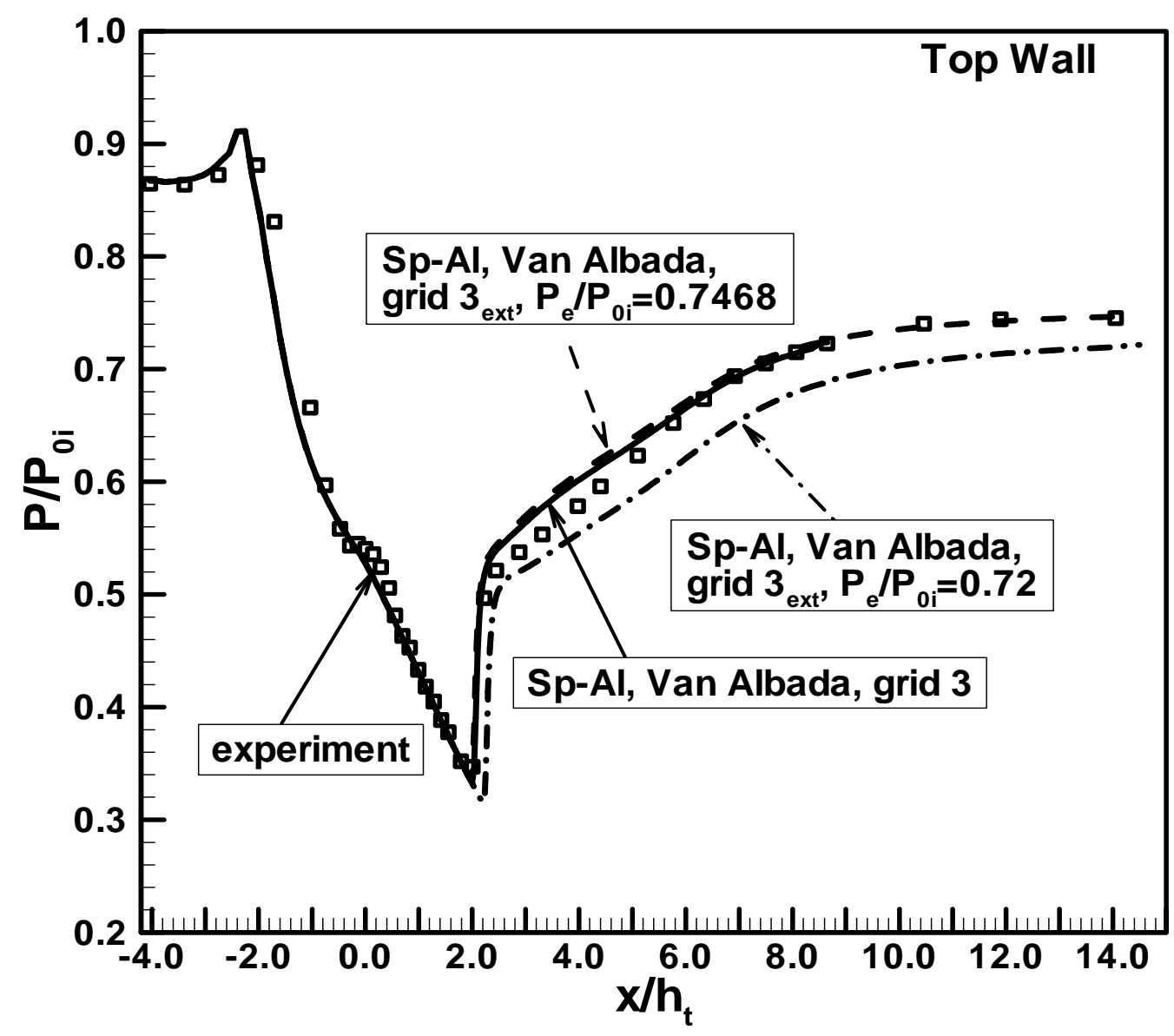

Figure 14: Top wall pressure distributions obtained with different versions of the diffuser geometry and exit pressure ratios for the strong shock case (the results of the Sp-Al model, Van Albada limiter, and Grids g3 and g3ext are shown). 